\title{
Solving Sequences of Refined Multistage Stochastic Linear Programs
}

\author{
Karl Frauendorfer, Gido Haarbrücker \\ Institute for Operations Research, University of St. Gallen, Switzerland
}

\begin{abstract}
Multistage stochastic programs with continuous underlying distributions involve the obstacle of high-dimensional integrals where the values of the integrands again are given by solutions of stochastic programs. A common solution technique consists of discretizing the support of the original distributions leading to scenario trees and corresponding LPs which are - up to a certain size - easy to solve. But in order to improve the accuracy of approximation, successive refinements of the support result in rapidly expanding scenario trees and associated LPs. Hence, the solvability of the multistage stochastic program is limited by the numerical solvability of sequences of such expanding LPs. This work describes an algorithmic technique for solving the large-scale LP of refinement $\nu$ based on the solutions at the previous $\nu-1$ refinements. Numerical results are presented for practical problem statements within financial applications demonstrating significant speedup (depending on the size of the LP instances).
\end{abstract}

Keywords: Discretization Schemes, Multistage Stochastic Linear Programs, Optimality Condition, Financial Applications, Barycentric Approximation 


\section{Introduction}

A huge number of practical problem situations can be formulated as an optimization problem, i.e. can be reduced to the question of maximizing or minimizing a certain quantity with respect to some constraints. Passing over to Multistage Stochastic Programming, additional important real-life characteristics like random data, dynamic evolution over time and multiple decision points can be incorporated into the problem formulation. Then, in general, the expected value of the interesting quantity will be maximized or minimized. In this paper, we confine ourselves to multistage stochastic programs with recourse for two reasons: firstly, these programs have proved to be an adequate tool reflecting the complexity of real-life situations; secondly, in the last decades a wide theory and powerful solution procedures have been developed. When stochastic influence is given by continuous distributions, one of the most common - and in fact promising - approaches is to discretize the support of these distributions. Of course, although we concentrate on discretization approaches, we do not wish to conceal the existence of alternative methods: for instance, approximation of the original distributions can also be achieved by simpler - but still continuous! - distributions (see e.g. Birge and Wallace [4], Birge and Wets [5], Wallace [22], and Birge and Qi [3]). Discretization always leads to scenario trees depicting stochastic evolution and thus overcomes the obstacle of evaluating high-dimensional integrals when determining expected values. Naturally, the solutions obtained consisting of (expected) objective values and optimal first-stage decisions are solutions of approximate programs: they can only serve as approximating solutions of the original 'continuously distributed' program.

Improvement of the approximation is usually achievable via successive refining of the support of the approximating discrete (conditional) probability measures. Each of these refinements increases considerably the size of the corresponding large-scale linear program. Performing many refinement steps thus leads to a sequence of ever enlarging LPs. In order to track the improvement of the approximations every enlarged LP has to be solved, requiring a great deal of time. Throughout this paper we will assume such single LPs to be solved via so-called direct methods, i.e. standard optimization methods which do not apply special large-scale strategies like e.g. decomposition or relaxation. In fact, the classic primal simplex algorithm was applied for the evaluation of the numerical results upcoming in section 6 . Readers interested in a brief survey on direct and alternative methods are referred to Birge [1].

Fortunately, there are practical problem situations which are characterized by a certain robustness of the optimal approximate first-stage decision $x^{1 \star}$ in the sense that $x^{1^{\star}}$ remains constant over a certain sequence of refinements. Hence, in such cases there is no need to optimize anew every increased LP as a whole. Instead, it would be sufficient to show the last obtained optimal $x^{1^{\star}}$ to be still optimal after one (or more) additionally realized refinements; e.g. it would be 
sufficient that $x^{1^{\star}}$ fulfills a classical optimality condition: the negative subgradient of the objective function at $x^{1^{\star}}$ is normal to the feasibility region of $x^{1}$ at $x^{1^{\star}}$. Provided the above-mentioned robustness and assuming a specific characteristic of the discretization scheme used, this verification of the sufficient optimality condition - replacing the optimization of an increased LP as a whole - should be a promising way to reduce numerical effort.

Run-time investigation in this paper will use the Barycentric Approximation scheme for generating scenario trees. But since the type of the discretization scheme (e.g., sampling leading to probabilistic bounds for the true optimal objective value or chosing special points out of the support leading to deterministic bounds) used is not central to the development of the theoretical framework concerning relevant (sub-)gradients or the optimality check, we postpone its brief treatment to section 5. Theoretical aspects are contained in section 2 and are based on a common notation of multistage stochastic problems with recourse to be found, e.g., in the stochastic programming textbook by Birge and Louveaux [2]. A description of a practical problem formulation in the area of running fixed income products which underlies the computation runs will be given in section 4 . Section 6 lists numerical results of the presented new approach w.r.t. computation time and speedup. The paper ends with conclusions and a brief outlook.

Note This paper summarizes major ideas from a doctoral thesis of one of the authors (Haarbrücker [14]) written at the University of St. Gallen, Switzerland.

\section{Theoretical Framework}

This section starts with an introduction of a multistage stochastic linear program with recourse, using a widespread notation which is different from that notation used in original articles (see e.g. Frauendorfer [7][8][9]) covering the Barycentric Approximation scheme itself. This is done for two reasons: firstly, at this point of progress, there will be neither formal nor logical connections to the Barycentric Approximation scheme; all considerations can be detached from the discretization method used; secondly, the notation used is a little more intuitive and hopefully simplifies the upcoming descriptions.

Throughout this paper, we deal with problems containing stochasticity in the objective function and in the RHS only, i.e. there are no random data in the constraint matrix. Section 3 states a general optimality condition out of nonlinear - and not at all stochastic - optimization. A detailed transfer of this condition to the previously stated multistage stochastic linear program (MSLP) is omitted. Instead, we sketch a formulation of an optimality check which is in line with the existing literature. The general aim will be to avoid the time-consuming optimization runs within the traditional solution approach where - when faced with a sequence of expanding scenario trees and associated linear programs - each of 
the successively occurring large-scale LPs is solved anew as a whole. Hence, optimization runs will be replaced by a kind of optimality check, where the latter allows us to expect reduced CPU times for solving a sequence of increased LPs arising from a discretization scheme. In subsection 2.2, we present an illustrative view of the requirements which have to be fulfilled in order to get this optimality check as a sensible alternative to the traditional solution approach. Finally, the last subsection describes the traditional approach of solving a sequence of increasing approximating programs and introduces a promising new approach. This will be done rather informally with a focus on verbal explanations; a more detailed treatment of key elements like (sub-)gradients or normal cones is postponed to section 3 .

\subsection{Multistage Stochastic Linear Program}

Using a dynamic programming type of recursion, we can write a MSLP in the following form:

for $t=H, \ldots, 2$ define backwards

$$
\begin{aligned}
Q^{t}\left(x^{t-1}, \boldsymbol{\xi}^{t}(\omega)\right):= & \min \quad \boldsymbol{c}^{t}(\omega) x^{t}(\omega)+E_{\boldsymbol{\xi}^{t+1}}\left[Q^{t+1}\left(x^{t}, \boldsymbol{\xi}^{t+1}(\omega)\right)\right] \\
\text { s.t. } \quad & W^{t} x^{t}(\omega)=\boldsymbol{h}^{t}(\omega)-T^{t-1} x^{t-1} \\
& x^{t}(\omega) \geq 0
\end{aligned}
$$

with terminal value function given by

$$
Q^{H+1}(\cdot, \cdot) \equiv 0 \text {. }
$$

The interesting minimal objective value and the today's optimal decision can then be obained by solving the problem

$$
\begin{array}{ll}
\min & z\left(x^{1}\right):=c^{1} x^{1}+\mathcal{Q}^{2}\left(x^{1}\right) \\
\text { s.t. } & W^{1} x^{1}=h^{1} \\
& x^{1} \geq 0 .
\end{array}
$$

In case of unboundedness below or infeasibility of the program $(1) Q^{t}\left(x^{t-1}, \boldsymbol{\xi}^{t}(\omega)\right)$ is set to $-\infty$ or $+\infty$, respectively. The expectation functional $\mathcal{Q}^{2}(\cdot)$ of the value function $Q^{2}(\cdot, \cdot)$ in $(2)$ is defined as

$$
\begin{aligned}
\mathcal{Q}^{2}\left(x^{1}\right) & :=E_{P^{\xi^{2}}}\left[Q^{2}\left(x^{1}, \cdot\right)\right]=\int_{\Xi^{2}} Q^{2}\left(x^{1}, \xi^{2}\right) d P^{\boldsymbol{\xi}^{2}}\left(\xi^{2}\right) \\
& =\int_{\Omega} Q^{2}\left(x^{1}, \boldsymbol{\xi}^{2}(\omega)\right) d P(\omega)=E_{P}\left[Q^{2}\left(x^{1}, \boldsymbol{\xi}^{2}\right)\right]
\end{aligned}
$$

with

$$
\begin{aligned}
Q^{2}\left(x^{1}, \boldsymbol{\xi}^{2}(\omega)\right):=\min & \boldsymbol{c}^{2}(\omega) x^{2}(\omega)+\mathcal{Q}^{3}\left(x^{2}\right) \\
\text { s.t. } & W^{2} x^{2}(\omega)=\boldsymbol{h}^{2}(\omega)-T^{1} x^{1} \\
& x^{2}(\omega) \geq 0 .
\end{aligned}
$$


Sometimes the expectation functionals $\mathcal{Q}^{t}$ are named expected value functions, too (see e.g. Kall and Wallace [16], p. 148). In the following, we refrain from a differentiation from 'normal' value functions; depending on the situation and notation it will become clear if it concerns the evaluation of an expectation or not.

In the above mathematical formulation, $\Xi^{2}$ denotes the support of $\boldsymbol{\xi}^{2}$; as to the definition of $P$ see below. Integrals in the form of $E_{P^{\xi^{2}}}[\cdot]$ are given as the sum of their positive and negative parts with positive (negative) parts to be defined as $+\infty(-\infty)$ when the integral diverges or the integrand takes the value $+\infty$ $(-\infty)$ on a set with strictly positive measure. ${ }^{1} c^{1}$ and $h^{1}$ are known vectors out of $\mathbb{R}^{n_{1}}$ and $\mathbb{R}^{m_{1}}$, respectively. For all stages $t=2, \ldots, H$ the random vectors $\boldsymbol{\xi}^{t}:=\left(\boldsymbol{c}^{t}, \boldsymbol{h}^{t}\right)$ fulfill

$$
\begin{aligned}
\boldsymbol{\xi}^{t}=\left(\boldsymbol{c}^{t}, \boldsymbol{h}^{t}\right):\left(\Omega, \Sigma^{t}\right) & \longrightarrow\left(\mathbb{R}^{n_{t}+m_{t}}, \mathcal{B}^{n_{t}+m_{t}}\right) \\
\omega & \longmapsto \boldsymbol{\xi}^{t}(\omega)=\left(\begin{array}{c}
\boldsymbol{c}^{t}(\omega) \\
\boldsymbol{h}^{t}(\omega)
\end{array}\right)=:\left(\begin{array}{c}
c^{t} \\
h^{t}
\end{array}\right)
\end{aligned}
$$

with $\Sigma^{t} \subseteq \Sigma^{t+1} \sigma$-algebras relative to $\Omega(t=2, \ldots, H-1)$; herein, (6) stands for the $\left(\Sigma^{t}, \mathcal{B}^{n_{t}+m_{t}}\right)$-measurability of $\boldsymbol{\xi}^{t}$ with $\mathcal{B}^{n}(n \in \mathbb{N})$ denoting the usual $\sigma$ algebra generated by all open subsets of $\mathbb{R}^{n}$. Each $\sigma$-algebra $\Sigma^{t}$ consists of those events that are 'known' at time $t$. Let $P$ be a known probability measure on the measurable space $\left(\Omega, \Sigma^{H}\right)$. Because of the monotone increasing - in the sense of set inclusion $-\sigma$-algebras $\Sigma^{t}$ all $\left(\Omega, \Sigma^{t}, P\right)$ are well-defined probability spaces for $t=2, \ldots, H$.

Concerning the decisions, we additionally demand the $\left(\Sigma^{t}, \mathcal{B}^{n_{t}}\right)$-measurability of $x^{t}$ for all $t$; this ensures the so-called nonanticipativity of the decisions to be taken, i.e. decisions made today cannot depend on information received tomorrow (or any day thereafter).

$x^{t-1}$ denotes the states of the system and $E_{P \xi^{t}}[\cdot]$ the expected value w.r.t. the induced probability measure $P^{\boldsymbol{\xi}^{t}}(t=2, \ldots, H)$. The value functions $Q^{t}(\cdot, \cdot)$ and expectation functionals $\mathcal{Q}^{t}(\cdot)$ of stages $t>2$ are defined analogously to $Q^{2}(\cdot, \cdot)$ and $\mathcal{Q}^{2}(\cdot)$, respectively; $\Xi^{t}$ designates the support of $\boldsymbol{\xi}^{t}$.

\section{Remark 2.1 :}

(i) The distribution of $\boldsymbol{\xi}^{t}$ is assumed to be independent of past realizations. If this assumption is given up, ${ }^{2}$ then the problem formulation must contain - apart from state information $x^{t-1}$ - further information in the form of

\footnotetext{
${ }^{1}$ Cases of simultaneous occurrence of $-\infty$ and $+\infty$ are treated by the convention $(-\infty)+\infty=$ $+\infty$ (see e.g. Wets [24], p. 312), i.e. infeasibility in one subproblem cannot be compensated by unboundedness below in another subproblem.

${ }^{2}$ Take note of the fact that relaxing the stochastic independence cannot be done without any compensation but only in favour of some weaker kind of regularity condition like, e.g., linear dependency (see Wets [23], pp. 202f, 205).
} 
observed realizations of the random vectors up to time $t$. Since the upcoming applications in section 4 are characterized by stochastic independence between stages, we refrain from including such further information in the formulation for ease of notation.

(ii) Both the technology matrices $T^{t}$ and the recourse matrices $W^{t}$ are known matrices with dimension $m_{t} \times n_{t}$. Because of its deterministic recourse matrices the model is named fixed recourse model. Hence, due to the deterministic technology matrices, all stochastic influence in (1) and (5) is restricted to the objective function and the RHS of the constraints. But it is left to the reader to assume stochastic influence on the technology matrices as well (with appropriate adjustments of $\boldsymbol{\xi}^{t}, \Xi^{t}$ and $\Sigma^{t}$ ), leading to the term $\boldsymbol{T}^{t}(\omega)$. Again with regard to the applications in section 4, throughout this paper all technology matrices are generally written in deterministic form.

(iii) For the integrals stated so far to be well defined, the random vectors $\boldsymbol{\xi}^{t}$ have to fulfill some further technical assumptions, because measurability of the value functions $Q^{t+1}\left(x^{t}, \boldsymbol{\xi}^{t+1}(\cdot)\right)$ cannot be a foregone conclusion (see Wets [23], p. 202). To ensure measurability, we therefore make two general assumptions which are sufficient to show the measurability of the $Q^{t+1}$ (Wets [23], p. 202): (a) existing variance for all random variables $\boldsymbol{c}_{i}^{t}$ and $\boldsymbol{h}_{k}^{t}$ (i.e. for all components of $\boldsymbol{\xi}^{t}$ ) on all stages $t$, and (b) the closure of the convex hull of the corresponding supports to be polyhedral convex, i.e. expressible as an intersection of some finite collection of closed half-spaces. Alternatively, the above-mentioned square-integrability of the components of $\boldsymbol{\xi}^{t}$ could be replaced by the demand for compact supports $\Xi^{t}$ for all $t$ (see Wets [23], Cor. 4.2). ${ }^{3}$

Given a continuous distribution of $\boldsymbol{\xi}^{t}$, one is faced with the problem of multidimensional integration since the value functions are in fact expectation functionals. A typical way to overcome this burden is the reduction of $\Xi^{t}$ to a finite number of states. At this point, it is of no interest which technique is used to discretize the supports $\Xi^{t}$. Whatever scheme is applied in order to determine states out of $\Xi^{t}$ and associated probabilities, the finite support of such approximating discrete probability measures leads to an alternative formulation of (5). Since this will often be used in the upcoming considerations, we state this formulation explicitly:

$$
\mathcal{Q}^{2}\left(x^{1}\right)=E_{\xi^{2}}\left[Q^{2}\left(x^{1}, \xi^{2}(\omega)\right)\right]=\sum_{s=1}^{S} p_{s}^{2} Q^{2}\left(x^{1}, \xi_{s}^{2}\right)
$$

\footnotetext{
${ }^{3}$ In fact, these prerequesites are a bit too strong as they cover even the case of stochastic technology matrices; then, of course, with the assumption extended to the components $\boldsymbol{T}_{k j}^{t}$ of $\boldsymbol{T}^{t}$.
} 
with

$$
\begin{aligned}
Q^{2}\left(x^{1}, \xi_{s}^{2}\right):=\min & c_{s}^{2} x_{s}^{2}+\mathcal{Q}^{3}\left(x_{s}^{2}\right) \\
\text { s.t. } & W^{2} x_{s}^{2}=h_{s}^{2}-T^{1} x^{1} \\
& x^{2} \geq 0
\end{aligned}
$$

Herein, $S$ is the total number of states in the second stage which are denoted by $\xi_{s}^{2}$; let the $p_{s}^{2}$ denote their associated probabilities.

\subsection{Successive Discretization}

Consider a scenario tree representing a deterministic equivalent program after having carried out a sequence of $\nu$ refinements of the support of the underlying probability distributions (where we confine ourselves to refinements in the root node only, i.e. partitioning always takes place w.r.t. the support of second-stage distributions). This restriction to refining in the root node is done for two reasons: firstly, this kind of refinement results in a largest possible increase of the number of scenarios and thus represents the most challenging case as the corresponding LPs enlarge to the same extent. Secondly, this fast growth of the scenario tree stands for a very exhaustive incorporation of potential dynamics of the uncertain quantities and can be assumed to result - to a certain extent, as we will see later on - in a strong improvement of the approximation accuracy; see Marohn [17], Chapter 5.

The upper part of Fig. 1 gives an exemplary image of such a refined scenario tree when the unrefined tree consisted of an ordinary binary tree. Having carried out a further refinement step $\nu+1$ (in the sense that the chosen part of the support will be split), the subtree contained in the upper framed box will vanish and has to be replaced by new subtrees representing the new discretization points. If, for ease of simplicity, we think of a simple split of the partial support into two pieces, then the new parts are given by the two lower dashed boxes. Take note that, naturally, one can obtain new subtrees which are of exactly the same form as the vanishing part (for instance, when each of the two new partial supports is represented by the same number of discretization points). We will come back to this aspect when dealing with the need to solve these new subproblems on the operational level by means of a commercial LP-solver. For every refinement step $\nu$, the scenario tree achieved is associated with a linear program $L P_{1}^{\nu}$ : subscript 1 indicates the stage of the root node and the superscript $\nu$ the number of refinements carried out so far. Considering now the second-stage problems $L P_{2, s}^{\nu}$ (with $s=$ $1, \ldots, S^{\nu}$ numbering both the second-stage nodes from left to right and reflecting the different states of the system on that stage, i.e. the specific discretization points of the support of $\boldsymbol{\xi}^{2}$ ), the next refinement step $\nu+1$ leads to the following situation in our specific example of Fig. 1: 


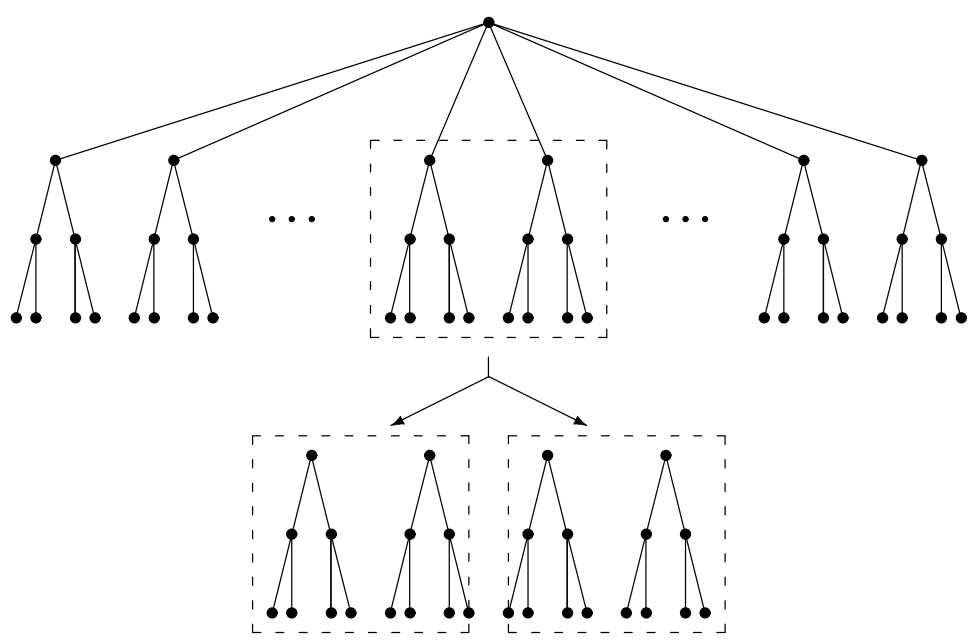

Figure 1: Changing parts of a scenario tree per refinement

- The overall linear program $L P_{1}^{\nu}$ is replaced by $L P_{1}^{\nu+1}$. The new number of second-stage nodes is given by $S^{\nu+1}:=S^{\nu}+2$.

- The two old second-stage subproblems, say $L P_{2, s^{\star}}^{\nu}$ and $L P_{2, s^{\star}+1}^{\nu}$, are replaced by $L P_{2, s^{\star}}^{\nu+1}, L P_{2, s^{\star}+1}^{\nu+1}, L P_{2, s^{\star}+2}^{\nu+1}$ and $L P_{2, s^{\star}+3}^{\nu+1}$.

- All the other old second-stage subproblems $L P_{2, s}^{\nu}, s \in\left\{1, \ldots S^{\nu}\right\} \backslash\left\{s^{\star}, s^{\star}+\right.$ $1\}$, remain unchanged and form the rest of the subproblems $L P_{2, s}^{\nu+1}$.

Usually, when using whatever solver to optimize the large-scale LPs, the traditional procedure is as follows:

\section{Traditional Approach}

1. Build up $L P_{1}^{0}$ and convey problem to the solver. Solve $L P_{1}^{0}$ and get optimal solution $x^{1,0}$. Set $\nu=1$.

2. Carry out refinement step $\nu$. Build up $L P_{1}^{\nu}$ and convey problem to the solver. Solve $L P_{1}^{\nu}$ and get optimal solution $x^{1, \nu}$. Set $\nu=\nu+1$.

3. IF $\nu<\operatorname{Max}^{4}$ and $x^{1, \nu}$ does not fulfill a certain prechosen stopping-rule GOTO 2. ELSE STOP.

Naturally, this approach can be modified in the sense that the LP is solved only after every $k^{\text {th }}(k>1)$ refinement. However, when the main interest is to track the improvement of single refinement steps or to stop as soon as the stopping criterion is fulfilled, one cannot avoid solving the large-scale LP associated with each

\footnotetext{
${ }^{4}$ Max indicating the maximal number of refinement steps
} 
refinement. Having in mind the so-called curse of dimensionality, the bottleneck of this approach obviously is given by solving these expanded large-scale LPs. It is this computational burden which will be overcome by the new approach, reducing the numerical effort by trying to show that the formerly obtained optimal solution is still optimal after the very last refinement step.

Keeping in mind the drawback of the traditional approach, the second and third topics of the previously mentioned list become important in two senses: firstly, they motivate the idea of reducing the solving of each refined problem as whole to a much easier solving of just the new smaller second-stage problems with a subsequent (still to be determined!) optimality check. Secondly, they reveal some rather heuristic properties that the refinement method used must necessarily possess: with ongoing refinement process, the replaced parts of the scenario tree have to become smaller and smaller relative to the whole scenario tree; additionally, all parts besides the replaced part have to remain totally unchanged. Otherwise the new approach of solving just subproblems and checking optimality cannot be expected to save much numerical effort and, hence, valuable computation time.

The necessity of these requirements becomes clearer when focussing on the optimality check itself: this is done in the following section, where we transfer a well-known optimality condition out of nonlinear optimization to the hereformulated MSLP.

\section{Conception and Implementation of a new Approach}

Having in mind that in many practical problem situations the multistage stochastic problem is periodically solved, it is the root node decision $x^{1}$ which is of main interest. Its associated costs are given by

$$
z=c^{1} x^{1}+\mathcal{Q}^{2}\left(x^{1}\right)
$$

where

$$
\mathcal{Q}^{2}\left(x^{1}\right)=\sum_{s=1}^{S} p_{s}^{2} Q^{2}\left(x^{1}, \xi_{s}^{2}\right) .
$$

In order to approach the formerly mentioned task of an optimality check, one needs a sufficient condition proving an $x^{1}$ to have minimum costs. The underlying idea can be stated quite generally with the following theorem containing a general optimality condition (see e.g. Rockafellar [18], p. 270):

Theorem 3.1:

Let $\varphi$ be a proper convex function, and let $C$ be a non-empty convex set. In order 
that $c$ be a point where the infimum of $\varphi$ relative to $C$ is attained, it is sufficient that there exists a vector $x^{\star} \in \partial \varphi(c)$ such that $-x^{\star}$ is normal to $C$ at $c$.

Hence, rewriting $z$ in (9) as a function of $x^{1}$, it is obvious that $z\left(x^{1}\right)$ is a rather natural choice for the function $\varphi(x)$ in Th. 3.1. For ease of exposition, we skip a formal mathematical treatment which would show that the function $z$ and its approximations $z_{\nu}$ (in a slightly reformulated manner) fulfill the assumptions of Th. 3.1.

Next, we introduce a new method as an alternative to the traditional approach stated at the end of the previous section. At this point of progress, the alterntive approach is presented in form of a meta-algorithm only: a course of modules is given with details hiding behind black-boxes termed, e.g., 'Evaluate' or 'Update'. In the following, the new method will be called progressive refinement approach.

\section{Progressive Refinement Approach (PRA)}

1. Set $\nu=0$.

2. Build up $L P_{1}^{\nu}$ and convey problem to the solver. Solve $L P_{1}^{\nu}$ and get optimal solution $x^{1, \nu}$.

IF $\nu=$ Max or $x^{1, \nu}$ fulfills a certain prechosen stopping-rule STOP.

3. Solve all $L P_{2, s}^{\nu}$ with $x^{1, \nu}$ fixed in the RHS. Evaluate (sub-)gradient $g^{\nu}$ of $z_{\nu}$ at $x^{1, \nu}$, i.e. in the notation of Th. 3.1 a $g^{\nu} \in \partial z_{\nu}\left(x^{1, \nu}\right)$.

4. Set $\nu=\nu+1$.

Carry out refinement step $\nu$. Build up new subproblems $L P_{2, s}^{\nu}$ and convey subproblems to the solver. Solve subproblems.

5. Update last evaluated (sub-)gradient $g^{\nu-1}$ to the current one $g^{\nu}$. Check optimality, i.e. try to show that $-g^{\nu} \in \mathcal{N}_{X^{1}}\left(x^{1, \nu-1}\right) .^{5}$

IF positive: Set $x^{1, \nu}=x^{1, \nu-1}$.

IF $\nu<$ Max and $x^{1, \nu}$ does not fulfill a certain prechosen

stopping-rule GOTO 4.

ELSE STOP.

ELSE (i.e. negative) GOTO 2.

\section{Remark 3.1 :}

(i) The advantage of the PRA is incorporated into steps 4 and 5: only the new second-stage subproblems (remember Fig. 1) have to be solved and an optimality check has to be done. This will lead to significant savings w.r.t. computation time for two reasons: first, the subproblems become

\footnotetext{
${ }^{5} \mathcal{N}_{X^{1}}\left(x^{1, \nu-1}\right)$ denotes the normal cone to the feasibility region $X^{1}$ of $x^{1}$ at the previously achieved optimal solution $x^{1, \nu-1}$.
} 
smaller and smaller relative to the size of the whole problem; secondly, there are always only a few subproblems to solve - on condition of adequate configuration of the discretization scheme used.

(ii) Of course, a tradeoff exists between the smaller computational effort of solving the subproblems and the additional effort of carrying out the optimality check itself. If the latter takes too much time, then on balance no overall time saving will be achieved.

(iii) Possible drawbacks are given by step 3: whenever the optimality of the former optimal decision $x^{1, \nu}$ could not be shown, in addition to solving anew the whole refined problem (see $(i v)$ ), all second-stage subproblems have to be optimized. Of course, with ongoing refinement, the number of these subproblems can become quite large and even amount to several hundred. Fortunately - as already mentioned in section 1 - in the special problem which will be treated here, we empirically observed that normally the root node decision remains constant over longer sequences of refinements. Hence, the case where one is forced to solve all subproblems does not occur that often.

(iv) When at an advanced phase of the refinement process (i.e. having carried out numerous refinement steps) optimality could not be shown, then one runs into the same trouble as happens within the traditional approach: the corresponding LP becomes too large to be solved with acceptable computational effort.

(In the following, the refinement index $\nu$ will be dropped for notational simplicity; the reader should keep in mind that functions $z, Q^{t}$ and $\mathcal{Q}^{t}$ stand for approximate functions of the true ones.) As can be found in the literature, see e.g. Birge and Louveaux [2], p. 129, a discrete support results in polyhedral convex value functions $\mathcal{Q}^{t}$. Obviously, this leads to an only piecewise continuous differentiability of the value functions $\mathcal{Q}^{t}$ and, hence, of the function $z$, too. For lack of space, we omit a formal case differentiation between the differentiable and the non-differentiable case and just summarize its results: when carrying out computations on the operational level, one can always evaluate the same vector $g$ without being forced to test for differentiability of $z$, namely

$$
g:=c^{1}-\sum_{s=1}^{S} p_{s}^{2}\left(\pi_{s}^{2}\right)^{\prime} T^{1} .
$$

Herein, $\pi_{s}^{2}$ denote the simplex multipliers in the optimum of the $s$-th second-stage subproblem. In the differentiable case the vector provides the (unique) gradient; in the strict subdifferentiable case, the same vector provides a special 'extreme' 
subgradient. ${ }^{6}$ Only in theoretical notations, one has to distinguish between the gradient

$$
g:=c^{1}-\sum_{s=1}^{S} p_{s}^{2}\left(\pi_{s}^{2}\right)^{\prime} T^{1}=\nabla z\left(x^{1}\right)
$$

in the differentiable case and the (specifically chosen) subgradient

$$
g=c^{1}-\sum_{s=1}^{S} p_{s}^{2}\left(\pi_{s}^{2}\right)^{\prime} T^{1} \in\left\{c^{1}\right\}+\sum_{s=1}^{S} p_{s}^{2} \partial\left(Q^{2}\left(\cdot, \xi_{s}^{2}\right)\right)\left(x^{1}\right) \subset \partial z\left(x^{1}\right) .
$$

in the non-differentiable case. (Similar concepts relying on the same principle to speed up convergence by means of subgradient approximation also appear in e.g. Ruszczyński and Swietanowski [20] and Ruszczyński [19]: these papers place emphasis on a regularized decomposition approach instead of successive refinements.) Concerning the task 'Update' in step 5 of the PRA, one has to evaluate the new partial derivatives $\frac{d}{d x_{i}^{1}}\left(Q^{2}\left(x^{1}, \xi_{s}^{2}\right)\right)$, i.e. the partial derivatives for those $s$ which denote new states introduced by the new refinement. The rest of the partial derivatives doesn't have to be evaluated anew, given that the associated subproblems remained unchanged. The same holds for the nondifferentiable case, where an obvious 'extreme' subgradient can be upgraded in exactly the same manner. Altogether, a convenient update-handling of the (sub-)gradient is possible during the refinement process.

The next section treats more detailed the optimality check in step 5 of the PRA and its concrete handling on the implementation level.

\subsection{Optimality Check in Detail}

So far, in (11)-(12) we have introduced the (sub-)gradient $g$ which will be used within the optimality check of the PRA, multiplied by -1 according to Th. 3.1. It remains to focus on the other crucial element of the optimality check, i.e. the abstract normal cone $\mathcal{N}_{X^{1}}\left(x^{1, \nu-1}\right)$. Dealing with the normal cone to a feasibility region, we have to become more formal concerning the the definition of the function $z$ to be minimized over a convex feasibility set. Let us recall the description of a subproblem at stage $t$ in an analogous form to (7)-(8), i.e.

$$
\mathcal{Q}^{t}\left(x^{t-1}\right)=E_{\boldsymbol{\xi}^{t}}\left[Q^{t}\left(x^{t-1}, \xi^{t}(\omega)\right)\right]=\sum_{s=1}^{S^{t}} p_{s}^{t} Q^{t}\left(x^{t-1}, \xi_{s}^{t}\right)
$$

\footnotetext{
${ }^{6} \mathrm{By}$ this concentration on a single subgradient in case of strict subdifferentiability, we lose some power of the sufficient condition in Th. 3.1: instead of the equivalent condition $\partial \varphi(c) \cap$ $-\mathcal{N}_{C}(c) \neq \emptyset$ we employ only a reduced version $\{g\} \cap-\mathcal{N}_{C}(c) \neq \emptyset$.
} 
with

$$
\begin{aligned}
Q^{t}\left(x^{t-1}, \xi_{s}^{t}\right):= & \min \\
& c_{s}^{t} x_{s}^{t}+\mathcal{Q}^{t+1}\left(x_{s}^{t}\right) \\
\text { s.t. } & W^{t} x_{s}^{t}=h_{s}^{t}-T^{t-1} x^{t-1} \\
& x_{s}^{t} \geq 0 .
\end{aligned}
$$

Furthermore, we define - as is usually done $-Q^{t}\left(x^{t-1}, \xi_{s}^{t}\right)$ in (14) to be $+\infty$ if the corresponding feasibility set $\left\{x_{s}^{t} \mid W^{t} x_{s}^{t}=h_{s}^{t}-T^{t-1} x^{t-1}, x_{s}^{t} \geq 0\right\}$ is empty. The feasibility set for $x^{1}$ in our minimization problem can then be written as the intersection of the first-stage (deterministic) constraint set

$$
K^{1}:=\left\{x^{1} \mid W^{1} x^{1}=h^{1}, x^{1} \geq 0\right\}
$$

and the second-stage feasibility set

$$
K^{2}:=\left\{x^{1} \mid \mathcal{Q}^{2}\left(x^{1}\right)<\infty\right\} .
$$

Although we name $K^{2}$ a second-stage feasibility set, it is in fact a future-stages' feasibility set up to time $H$ because of the definition of the function $\mathcal{Q}^{2}$ : the recursive definition in (13) and (14) provides that $\mathcal{Q}^{2}$ is $\infty$, whenever the given decision $x^{1}$ cannot be feasibly extended up to time horizon $H$ for all scenarios (under the assumptions of all discrete probabilities $p_{s}^{t}>0$ - otherwise reduce the number of states - and the original problem to be bounded, which is actually a sensible assumption rather than a restrictive one). Using these definitions, we can rewrite the minimization problem with $z(\cdot)$ of $(9)$ in a more detailed way as

$$
\begin{aligned}
& \min z\left(x^{1}\right):=c^{1} x^{1}+\mathcal{Q}^{2}\left(x^{1}\right) \\
& \text { s.t. } x^{1} \in K^{1} \cap K^{2} \text {. }
\end{aligned}
$$

Determining $K^{2}$ is a very difficult matter: because of the transition of $x^{1}$ into the future by the technology matrices $T^{t}$, all future stochastic RHS parts $h_{s}^{t}$ can influence today's feasibility set; hence, no simple way of determining $K^{2}$ seems to be obvious. Fortunately, from a theoretical point of view, it is not absolutely necessary to have a complete description of the region of finiteness $K^{2}$ :

Knowing by definition of $K^{2}$ that $\mathcal{Q}^{2}\left(x^{1}\right)=\infty$ if and only if $x^{1} \notin K^{2}$, we can rewrite (17) as

$$
\begin{aligned}
\min & z\left(x^{1}\right) \\
& \text { s.t. } \quad x^{1} \in K^{1} .
\end{aligned}
$$

Hence, we can circumvent the need for a complete description of $K^{2}$ and can concentrate on the normal cone to the feasibility region $K^{1}$ for $x^{1}$. But we should be aware that this implicit use of our knowledge $\mathcal{Q}^{2}\left(x^{1}\right)=\infty \Leftrightarrow x^{1} \notin K^{2}$ expands 
the constraint region from $K^{1} \cap K^{2}$ to $K^{1}$ and thus reduces the corresponding normal cone: this becomes clear regarding the property ${ }^{7}$

$$
\mathcal{N}_{K^{1} \cap K^{2, \nu}}\left(x^{1}\right)=\mathcal{N}_{K^{1}}\left(x^{1}\right)+\mathcal{N}_{K^{2, \nu}}\left(x^{1}\right)
$$

which shows that using the program formulation (18) disregards the additive set component $\mathcal{N}_{K^{2, \nu}}\left(x^{1}\right)$ in (19) by just replacing it by the simple set $\{0\}$. Therefore, this relaxation can possibly entail cases where a previously evaluated optimal decision $x^{1}$ will not be shown to be still optimal after the very last refinement step - although in fact it is. Nevertheless, having in mind the difficulties of determining the second-stage feasibility set, we accept this relaxation: it will be seen in section 6 that such 'misleading' optimality checks occur very rarely.

Recalling (15), the normal cone to the feasibility set $K^{1}$ at a point $x^{1^{\star}} \in K^{1}$ is given by

$$
\mathcal{N}_{K^{1}}\left(x^{1^{\star}}\right)=\operatorname{cone}(A \cup B)
$$

with

$$
\begin{aligned}
A & :=\left\{ \pm\left(W_{i \bullet}^{1}\right)^{\prime} \mid i=1, \ldots, m_{1}\right\} \\
B & :=\left\{-e_{j} \mid x_{j}^{1^{\star}}=0, j=1, \ldots, n_{1}\right\}
\end{aligned}
$$

where $W_{i \bullet}^{1}$ denotes the $i^{\text {th }}$ row of $W^{1}, e_{j}$ the $j^{\text {th }}$ canonical unit vector in $\mathbb{R}^{n_{1}}$ and $x_{j}^{1^{\star}}$ the $j^{\text {th }}$ entry of the vector $x^{1^{\star}}$. To summarize, recalling Th. 3.1, (11), (12), and (20), we check the optimality condition

$$
-c^{1}+\sum_{s=1}^{S} p_{s}^{2}\left(\pi_{s}^{2}\right)^{\prime} T^{1} \stackrel{?}{\in} \mathcal{N}_{K^{1}}\left(x^{1^{\star}}\right)=\text { cone }(A \cup B) .
$$

A reader familiar with optimality conditions in the area of stochastic linear programs with recourse will easily verify that condition (21) is in line with results stated in the literature: see e.g. Birge and Louveaux [2], Section 3.1.e, or Wets [25], pp. $596 \mathrm{ff}$.

\section{Remark 3.2 :}

On the operational level, there exist several possibilities to check condition (21). One way is given by directly checking if the negative (sub-)gradient $-g$ lies in the normal cone. Hence, it is to check if the negative (sub-)gradient can be written as a non-negative linear combination of the column vectors in $A$ and $B$, i.e. if a

\footnotetext{
${ }^{7}$ In the approximating programs the sets $K^{2, \nu}$ and $K^{1}$ are polyhedral convex, so that under the sensible assumption $K^{1} \cap K^{2, \nu} \neq \emptyset$ (otherwise, the program would be infeasible) this property follows from e.g. Rockafellar [18], Cor. 23.8.1.

${ }^{8}$ cone $\left(\left\{a_{1}, \ldots, a_{n}\right\}\right):=\left\{\sum_{i=1}^{n} \lambda_{i} a_{i} \mid \lambda_{i} \geq 0, i=1, \ldots, n\right\}$ indicates the convex cone generated by $\left\{a_{1}, \ldots, a_{n}\right\}$.
} 
$\lambda:=\left(\lambda_{1}, \ldots, \lambda_{n}\right)^{\prime} \geq 0$ exists with $-g=[A B] \lambda$ (where $[A B]$ denotes the matrix containing as columns all vectors in $A$ and $B)$ ?

This can be done by simply solving a LP with equality and non-negativity constraints and an arbitrary objective function. Of course, it is not the optimal solution itself which is of interest when solving the LP; it is rather the question whether the LP is feasible or not: having found a basic feasible solution, this solution provides a $\lambda$ forming $-g$ as non-negative linear combination, as was stated above. Since commercial LP solvers (like e.g. CPLEX) do not always provide a special feasibility check, one can be forced to call the usual optimization routine. But anyway, as is well known, testing for feasibility is of the same complexity as solving a LP; hence, the optimization should not result in a much longer computation time compared to a feasibility check unless an unnecessarily complex objective function has been chosen. However, as long as the matrix $\left[\begin{array}{ll}A & B\end{array}\right]$ is not too large, the optimization should be realizable without much computational effort.

Another way of verifying if the negative (sub-)gradient $-g$ lies in the normal cone is given as follows: check whether the (sub-)gradient $g$ has non-positive inner products with all vectors in cone $(A \cup B)$, in particular with its generators. In order to do so, one would have to determine the polar matrix $[A B]^{\star}$ containing as columns all generators of the polar cone pol cone $\left[\begin{array}{ll}A & B\end{array}\right]:=\left\{u \mid u^{\prime} h \leq\right.$ 0 for all $h \in$ cone $[A B]\}$. An algorithm for determining these generators can be found in e.g. Kall and Wallace [16], p. 253ff.

In the next section, we describe a MSLP aimed at running financial products in an efficient manner. This MSLP has been developed in cooperation with a major Swiss bank and is used at monthly intervals to evaluate an efficient funding strategy of non-fixed rate mortgages. It will underlie all upcoming considerations and will be used to test the PRA.

\section{Financial Problem Statement}

In the following, we give a detailed description for the problem of funding nonfixed rate mortgages. Consider a bank which has to refinance loans given to the customers by taking up money of different maturities in the money market. This leads to a structure of maturing funds which is current today, and every time (from now up to some time horizon $H$ ) when an amount of the liability structure matures, this amount again has to be refinanced.

Interest rates and funding volume change stochastically over time. (The stochasticity of the latter is a specialty of Swiss non-fixed rate mortgages: mortgage takers are allowed to switch from non-fixed rate to fixed rate mortgages and vice versa; of course, they act depending on the current interest rate level. In general, a six months' notice holds for non-fixed rate mortgages.) The economic 
aim is a funding strategy for today minimizing the expected present value of the overall refinancing costs from today up to period $H$. Decisions have to be made at time points $t=1, \ldots, H$. At $t=1$ only the current interest rate curve and the structure of maturing funds $v_{o}^{d}$ are known. $\eta_{t}$ is a vector of risk factors which affect the interest rates at time $t$, and $\xi_{t}$ denotes the uncertain change in the funding volume at $t$. Dependent on $\eta_{t}$, the discounted accrued interest payments for refinancing $\$ 1$ in maturity $d$ at time $t$ are given by $\varphi_{t}^{d}\left(\eta_{t}\right)$. $\mathcal{D}:=\{1, \ldots, 12\}$ denotes a set of maturities in months; let $\mathcal{D}^{\text {liq }} \subset \mathcal{D}$ be the set of standard maturities for which the money market is sufficiently liquid. $x_{t}^{d}$ indicates the amount of borrowing at time $t$ with a maturity of $d$ months $\left(d \in \mathcal{D}^{\text {liq }}\right)$; the total volume of borrowings at time $t$ with a remaining $d$-month maturity $v_{t}^{d}(d \in \mathcal{D})$ is determined by

$$
\begin{array}{rlrl}
v_{t}^{d}-v_{t-1}^{d+1}-x_{t}^{d} & =0 & t=1, \ldots, H, d \in \mathcal{D}^{l i q} \\
v_{t}^{d}-v_{t-1}^{d+1} & =0 & t=1, \ldots, H, d \in \mathcal{D} \backslash \mathcal{D}^{l i q}
\end{array}
$$

At each time $t$, the overall funding volume $v_{t}$ must equal the sum (over all $d$ ) of existing borrowings with a remaining $d$-month maturity, i.e.

$$
v_{t}=\sum_{d \in \mathcal{D}} v_{t}^{d} \quad t=1, \ldots, H
$$

The funding volume at $t v_{t}$ is given by the funding volume in the previous period $t-1$ increased by the stochastic volume change $\xi_{t}$ :

$$
v_{t}=v_{t-1}+\xi_{t} \quad t=2, \ldots, T
$$

With $P$ denominating the joint multidimensional probability distribution of the underlying stochastic process $\left(\boldsymbol{\eta}_{t}, \boldsymbol{\xi}_{t}\right)$ the multistage stochastic problem reads as

$$
\begin{array}{rlrl}
\min \int \sum_{t=1}^{H} \sum_{d \in \mathcal{D}^{l i q}} \varphi_{t}^{d}\left(\eta_{t}\right) \cdot x_{t}^{d} d P(\eta, \xi) & \\
v_{t}^{d}-v_{t-1}^{d+1}-x_{t}^{d}=0 & t=1, \ldots, H, \forall d \in \mathcal{D}^{l i q} \\
v_{t}^{d}-v_{t-1}^{d+1} & =0 & t=1, \ldots, H, \forall d \in \mathcal{D} \backslash \mathcal{D}^{l i q} \\
v_{t}-\sum_{d \in \mathcal{D}} v_{t}^{d}=0 & t=1, \ldots, H \\
v_{t}-v_{t-1}=\xi_{t} & t=2, \ldots, H \\
v_{t}, v_{t}^{d} \in \mathbb{R} \text { nonanticipative } & t=1, \ldots, H, \forall d \in \mathcal{D} \\
& x_{t}^{d} \geq 0 \text { nonanticipative } & t & =1, \ldots, H, \forall d \in \mathcal{D}^{l i q} .
\end{array}
$$

The above-stated problem can be extended by introducing upper and lower limits for the amount of borrowing at time $t$ with a maturity of $d$ months, $x_{t}^{d}\left(d \in \mathcal{D}^{\text {liq }}\right)$. For ease of exposition, we omit this extension. A more detailed treatment of this special funding problem can be found in Frauendorfer/Haarbrücker [10], where 
a main focus lies on the underlying stochastic processes driving the dynamic evolution of interest rates and of the total funding volume.

Assuming $\boldsymbol{\eta}_{t}$ and $\boldsymbol{\xi}_{t}$ to be continuously distributed with supports $\Theta_{t}$ and $\Xi_{t}$, respectively, for all $t=1, \ldots, H$ and choosing finitely many points out of these two supports (in order to approximate the original problem), we can rewrite the above problem in the form of a scenario tree. Automatically, a scenario tree implies the required nonanticipativity so that the large-scale LP associated with the scenario tree contains nonanticipativity conditions only in an implicit way. Hence, extracting the recourse matrix $W^{1}$ and the technology matrix $T^{1}$ out of the above-stated multistage stochastic problem is straightforward and is only stated in brief in the next subsection.

\subsection{Recourse and Technology Matrix}

Taking a closer look at the above-stated constraints, it can easily be seen that the recourse matrix $W^{1}$ is of the following shape:

$$
W^{1}=\left[\begin{array}{c|c}
\left(-e_{j} \in \mathbb{R}^{D_{\max }}: j \in \mathcal{D}_{\leq}^{l i q}\right) & \boldsymbol{I}_{D_{\max }} \\
\hline 0 & -\mathbf{1}_{D_{\max }}^{\prime} \\
\hline \mathbf{1}_{D_{\max }}^{\prime} & 0
\end{array}\right]
$$

where $D_{\max }:=\max \{d \mid d \in \mathcal{D}\}$ indicates the maximum maturity and $\mathcal{D}_{\leq}^{\text {liq }}$ denominates $\mathcal{D}^{\text {liq }}$ increasingly ordered according to the total order $\leq$. $\boldsymbol{I}_{n}$ and $\mathbf{1}_{n}(n \in \mathbb{N})$ denote the $n$-dimensional identity matrix and the $n$-dimensional 1 -vector, respectively. The technology matrix $T^{1}$ is even simpler and given by

$$
T^{1}=\left[\begin{array}{c|c|ccc} 
& 0 & & & \\
& \vdots & & & \\
& \vdots & & -\boldsymbol{I}_{\left|\mathcal{D}^{l i q \mid}\right|} & \\
& 0 & & & \\
\cline { 2 - 6 } & 0 & 0 & \cdots & 0 \\
& 0 & 0 & \cdots & 0 \\
\cline { 2 - 5 } & -1 & 0 & \cdots & 0
\end{array}\right] .
$$

The left zero block is caused by the fact that tomorrow's effects of today's decisions are taken into account by the state variables $v_{1}^{d}$, and not by the decision variables $x_{1}^{d}$. Hence, recalling $\left(\pi_{s}^{2}\right)^{\prime} T^{1}$ to be a part of the overall (sub-)gradient in (11),(12), we get

$$
\left(\pi_{s}^{2}\right)^{\prime} T^{1}=-(\underbrace{0, \ldots, 0}_{\left.\right|^{\text {liq }}}, \pi_{s, D_{\max }+2}^{2}, \pi_{s, 1}^{2}, \ldots, \pi_{s, D_{\max }-1}^{2}),
$$

where $\pi_{s, i}^{2}$ denotes the dual variable corresponding to row $i$ once the second-stage subproblem $s$ has been optimized. Since in the mortgage problem statement 
only the first-stage decision variables $x_{1}^{d}\left(d \in \mathcal{D}^{l i q}\right)$ have deterministic objective function coefficients $>0$, the cost vector $c^{1}$ forming the inner product $c^{1} x^{1}$ in $(11),(12)$ is given by

$$
\begin{aligned}
c^{1} & :=(c_{\mathcal{D}_{\leq}^{l i q}}^{1}, \underbrace{0, \ldots, 0}_{D_{\max }}) \\
& :=\left(\left(\varphi_{1}^{d}\left(\eta_{1}\right): d \in \mathcal{D}_{\leq}^{l i q}\right), 0_{D_{\max }}\right) .
\end{aligned}
$$

To summarize, (24) and (25) lead to the following (sub-)gradient $g$ in a general notation (again, recall (11) - (12)):

$$
\begin{aligned}
g & =c^{1}-\sum_{s=1}^{S} p_{s}^{2}\left(\pi_{s}^{2}\right)^{\prime} T^{1} \\
& =\left(c_{\mathcal{D}_{\leq}^{l i q}}^{1}, 0_{D_{\max }}\right)+\sum_{s=1}^{S} p_{s}^{2}\left(0_{\left|\mathcal{D}^{l i q}\right|}, \pi_{s, D_{\max }+2}^{2}, \pi_{s, 1}^{2}, \ldots, \pi_{s, D_{\max }-1}^{2}\right) \\
& =\left(\left(\varphi_{1}^{d}\left(\eta_{1}\right): d \in \mathcal{D}_{\leq}^{l i q}\right), 0_{D_{\max }}\right)+\sum_{s=1}^{S} p_{s}^{2}\left(0_{\left|\mathcal{D}^{l i q}\right|}, \pi_{s, D_{\max }+2}^{2}, \pi_{s, 1}^{2}, \ldots, \pi_{s, D_{\max }-1}^{2}\right)
\end{aligned}
$$

\section{Remark 4.1 :}

It should be noted that not all components of the (sub-)gradient $g$ are in fact important for checking the containing-condition in (21). This can be seen as follows: the normal cone in (20) is now given by

$$
\text { cone }[A B]=\text { cone }\left[W^{1^{\prime}}-W^{1^{\prime}} B\right] \subset \mathbb{R}^{n_{1}}
$$

with $B$ again denoting the matrix of those canonical unit vectors corresponding to the non-negativity constraints which are fulfilled with "=". The special structure of $W^{1}$ (see (22)) now leads to the fact that cone $[A B]$ contains a whole subspace of $\mathbb{R}^{n_{1}}$. Hence, when one tries to form the negative (sub-)gradient $-g$ as a positive linear combination of the columns of $\left[W^{1^{\prime}}-W^{1^{\prime}} B\right]$, those components of $-g$ associated with the above-mentioned subspace are not restrictive at all: this fact can be shown using permutation matrices and orthogonal projectors, but we will omit its formal elaboration in this paper. However, taking into account this fact, the check of the condition in (21) can be written as

$$
\left(\begin{array}{c}
-c^{1^{\prime}} \mathcal{D}_{\leq}^{l i q} \\
-\sum_{s=1}^{S} p_{s}^{2} \pi_{s, D_{\max }+2}^{2} \\
-\left(\sum_{s=1}^{S} p_{s}^{2} \pi_{s, d-1}^{2}: d \in\left(\mathcal{D}^{l i q} \backslash\{1\}\right)_{\leq}\right)^{\prime}
\end{array}\right)
$$




$$
\stackrel{?}{\in} \quad \text { cone }\left[p r_{2\left|\mathcal{D}^{l i q}\right|}^{n_{1}}\left\{Q^{\prime} W^{1^{\prime}} P^{\prime}\right\}, p r_{2 \mid \mathcal{D}^{l i q \mid}}^{n_{1}}\left\{-Q^{\prime} W^{1^{\prime}} P^{\prime}\right\}, p r_{2 \mid \mathcal{D}^{l i q \mid}}^{n_{1}}\{B\}\right]
$$

where $Q, P$ are properly-chosen permutation matrices, i.e. square matrices with exactly one 1 in each row and each column and 0 anywhere else. $\{\cdot\}$ denotes the set of all columns of the enclosed matrix, and $\operatorname{pr}_{2 \mid \mathbb{D}^{l i q \mid}}^{n_{1}}(\cdot)$ denominates the orthogonal projector onto the first $2\left|\mathcal{D}^{l i q}\right|$ components of vectors in the $\mathbb{R}^{n_{1}}$. Computing the matrix products in (28) and applying the projection operator leads to the following much more simplified question:

$$
\begin{aligned}
& \exists \lambda \in \mathbb{R}_{\oplus}{ }^{n}:\left(\begin{array}{c}
-c^{1^{\prime}}{ }_{\mathcal{D}_{\leq}^{l i q}} \\
-\sum_{s=1}^{S} p_{s}^{2} \pi_{s, D_{\max }+2}^{2} \\
-\left(\sum_{s=1}^{S} p_{s}^{2} \pi_{s, d-1}^{2}: d \in\left(\mathcal{D}^{l i q} \backslash\{1\}\right)_{\leq}\right)^{\prime}
\end{array}\right)
\end{aligned}
$$

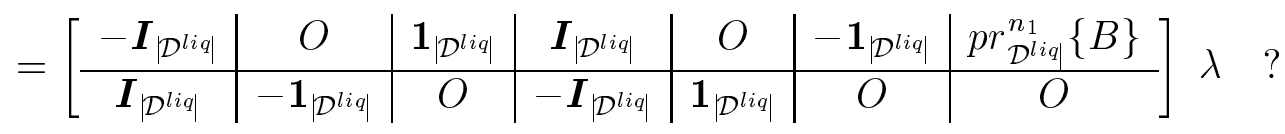

In this, the special shape of the last vertical block $\left[\frac{p r_{\left|D^{l i q}\right|}^{n_{1}}\{B\}}{O}\right]$ stems from the following fact: matrix $B$ is of the form

$$
B=\begin{array}{|c|}
X \\
\hline O_{D_{\max }}
\end{array}\left|\mathcal{D}^{l i q}\right|
$$

because non-negativity constraints only exist for the $\left|\mathcal{D}^{\text {liq }}\right|$ decision variables $x_{1}^{d}\left(d \in \mathcal{D}^{\text {liq }}\right)$, but not for the $D_{\text {max }}$ state variables $v_{1}^{d}(d \in \mathcal{D})$.

Actually, it is this condition (29) of remark 4.1 which is translated into a small LP (recall remark $3.2(\mathrm{ii})$ ); the latter is then conveyed to CPLEX and - using an arbitrary but simple objective function - solved by the ordinary primal simplex routine in order to check for feasibility.

So far, we have elaborated the relevant parts of the (sub-)gradient and the normal cone $\mathcal{N}_{K^{1}}\left(x^{1^{\star}}\right)$ which we need - in the framework of the special funding problem presented in section 4 - to carry out steps 3 and 5 of the PRA introduced in section 3. As was already mentioned in section 2.2, in general each refinement method fulfilling some requirements should be applicable in conjunction with the suggested new approach. We will concentrate solely on the barycentric approximation scheme for two reasons: firstly, it provides an appropriate discretization 
technique, given that the multistage stochastic problem has certain special properties; secondly, in our experience it has turned out to be an adequate technique in order to solve periodically the real-life problem of refinancing non-fixed rate mortgages. In the next section, we take a brief look at barycentric approximation itself.

\section{Barycentric Approximation Scheme}

We do not claim to give an exhaustive description of this special approximation scheme. We rather intend to clarify the relations of the discretization technique, the scenario trees generated and associated large-scale linear programs. For a detailed overview of the method, the interested reader is referred to Frauendorfer [7][8][9]. A compact treatment of the topic can be found e.g. in Schürle [21]; for a rather general formal exposition see Gaese [12]. A special treatment of refinement issues within the barycentric approximation scheme is given in Frauendorfer/Marohn [11] and Marohn [17]. For an illustrative approach concerning generalized barycenters - playing a key role in the barycentric discretization technique - see e.g. Haarbrücker [13]. An overview of the methodological idea for the 2 -stage case can be found in e.g. Kall and Wallace [16]. The reader familiar with barycentric approximation may skip this section and proceed with the numerical results set out in the next section.

Let $\Theta_{t} \subset \mathbb{R}^{K}$ and $\Xi_{t} \subset \mathbb{R}$ be regular simplices covering the support of $\eta_{\mathbf{t}}$ and $\xi_{\mathbf{t}}$ (recall section 4). Choosing so-called generalized barycenters of the $\times$-simplex ${ }^{9}$ $\Theta_{t} \times \Xi_{t}$ (see e.g. Frauendorfer [8]) as representatives of the support leads to upper and lower approximations (see Kall et al. [15], pp. $42 \mathrm{ff}$ ) of the saddle-structured value functions $Q^{t+1}(\cdot, \cdot)$. The size of the associated lower and upper approximating scenario trees depends on the dimensions of the regular simplices $\Theta_{t}$ and $\Xi_{t}$, respectively. In our case, these dimensions are $\operatorname{dim} \Theta_{t}=K$ and $\operatorname{dim} \Xi_{t}=2$ for all $t$. This leads to approximating (unrefined) lower and upper scenario trees with $\operatorname{dim} \Theta_{t}+1=K+1$ and $\operatorname{dim} \Xi_{t}+1=1+1=2$ sons per node, as well as $\frac{(K+1)^{H}-1}{K}$ and $2^{H}-1$ nodes in total, respectively.

Now, successive refinement of a tree in the root node means splitting one $\times$ simplex $\Theta_{2} \times \Xi_{2}$ into two sub- $\times$-simplices: Fig. 2 illustrates this situation where an $\times$-simplex $\Theta \times \Xi \subset \mathbb{R}^{2} \times \mathbb{R}$ is split (orthogonally) in two exemplary ways. The successive partitioning of the effective domains can be done in various ways. In the following, we focus only on the partitioning of $\Theta_{2} \times \Xi_{2}$ covering the support of the second-stage random vectors; i.e. the scenario tree is partitioned in the root node. Altogether, one meets the requirement already shown in Fig. 1: one part of the tree is replaced by two parts, each having exactly the same form as the vanishing part.

\footnotetext{
${ }^{9} \times$-simplex is short for cross simplex, the cartesian product of two simplices.
} 

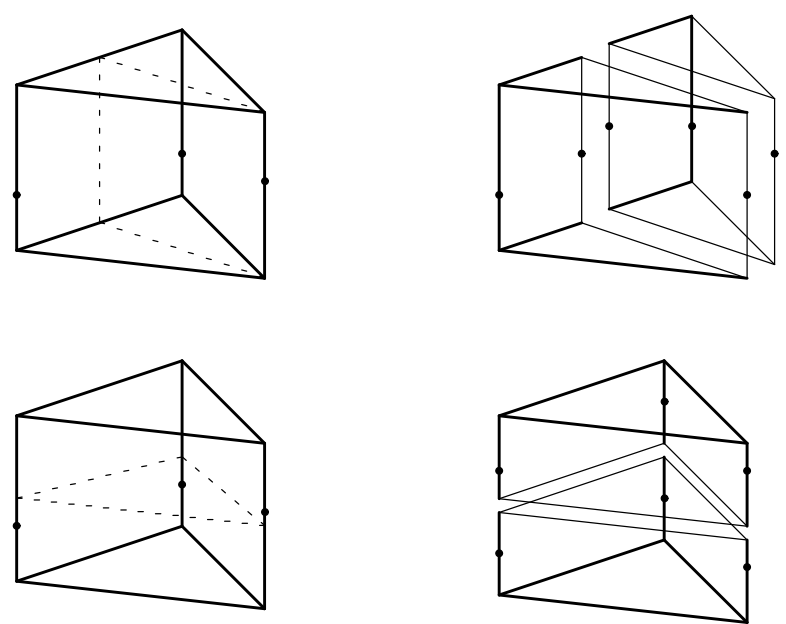

Figure 2: Splitting an $\times$-simplex with generalized barycenters of $\xi$ w.r.t. the vertices of $\Theta$

Naturally, the above-stated enlargement of the two approximating scenario trees results in a similar enlargement of the associated large-scale linear programs, since each new node leads to a new set of variables and constraints. (Just those constraints given in (4), but no additional nonanticipativity constraints since these are implicitly given by the tree structure.) In order to give an impression of the order of magnitude of large-scale LPs to be solved, Table 1 specifies the size of an LP corresponding to an unrefined lower scenario tree and of an LP corresponding to a lower tree after 4 refinements in the root node, i.e. - in the notation of section 2.2 - the size of $L P^{1,0}$ and $L P^{1,4}$, respectively, is reported (for $|\mathcal{D}|=D_{\max }=12$ and $\left.\left|\mathcal{D}^{l i q}\right|=5\right)$.

\section{$6 \quad$ Numerical Results}

This section serves to investigate the applicability of the PRA stated in section 3: for this purpose, a comparison of run-time performance both of the traditional and the new approach is listed, based on the financial application presented in section 4. Basic conditions concerning the run-time evaluation are stated in subsection 6.1. All original MSLP are successively refined in the root node according to the Barycentric Approximation scheme of section 5 and solved by the respective approach, i.e. by the traditional or PRA approach, respectively.

In order to cover the variety of real life constellations, we survey different structures of maturing volumes, time horizons, initial interest rate curves and number of stochastic variables influencing the interest rate dynamics. Detailed results concerning the funding of mortgages are listed in subsection 6.2. 


\begin{tabular}{|c|l||r|r|r|r|}
\hline \multicolumn{2}{|c||}{0 Refinements } & $\mathrm{H}=5$ & $\mathrm{H}=6$ & $\mathrm{H}=7$ & $\mathrm{H}=8$ \\
\hline \multirow{3}{*}{$\operatorname{dim} \Theta_{t}=K=2$} & rows & $1^{\prime} 815$ & $5^{\prime} 460$ & $16^{\prime} 395$ & $49^{\prime} 200$ \\
\cline { 2 - 6 } & columns & $2^{\prime} 178$ & $6^{\prime} 552$ & $19^{\prime} 674$ & $59^{\prime} 040$ \\
\cline { 2 - 6 } & nonzeros & $6^{\prime} 050$ & $18^{\prime} 200$ & $54^{\prime} 650$ & $164^{\prime} 000$ \\
\hline \hline \multirow{3}{*}{$\operatorname{dim} \Theta_{t}=K=3$} & rows & $5^{\prime} 115$ & $20^{\prime} 475$ & $81^{\prime} 915$ & $327^{\prime} 675$ \\
\cline { 2 - 6 } & columns & $6^{\prime} 138$ & $24^{\prime} 570$ & $98^{\prime} 298$ & $393^{\prime} 212$ \\
\cline { 2 - 6 } & nonzeros & $17^{\prime} 050$ & $68^{\prime} 250$ & $273^{\prime} 050$ & $1^{\prime} 092^{\prime} 250$ \\
\hline \hline \multirow{2}{*}{$\operatorname{dim} \Theta_{t}=K=2$} & $\mathrm{H}=5$ & $\mathrm{H}=6$ & $\mathrm{H}=7$ & $\mathrm{H}=8$ \\
\hline \multirow{2}{*}{$\operatorname{dim} \Theta_{t}=K=3$} & rows & $9^{\prime} 015$ & $27^{\prime} 240$ & $81^{\prime} 915$ & $245^{\prime} 940$ \\
\cline { 2 - 6 } & columns & $10^{\prime} 818$ & $32^{\prime} 688$ & $98^{\prime} 298$ & $295^{\prime} 128$ \\
\cline { 2 - 6 } & nonzeros & $30^{\prime} 050$ & $90^{\prime} 800$ & $273^{\prime} 050$ & $819^{\prime} 800$ \\
\hline & rows & $2^{\prime} 515$ & $102^{\prime} 315$ & $409^{\prime} 515$ & $1^{\prime} 638^{\prime} 315$ \\
\cline { 2 - 6 } & columns & $30^{\prime} 618$ & $122^{\prime} 778$ & $491^{\prime} 418$ & $1^{\prime} 965^{\prime} 978$ \\
\cline { 2 - 6 } & nonzeros & $85^{\prime} 050$ & $341^{\prime} 050$ & $1^{\prime} 365^{\prime} 050$ & $5^{\prime} 461^{\prime} 050$ \\
\hline
\end{tabular}

Table 1: Problem sizes corresponding to the lower tree for different horizons $H$ and dimensions of $\Theta_{t}$

\subsection{Basic Conditions}

The hardware platform comprised a Sun Ultra 1 workstation with 256 MB RAM and UltraSparc-IIi 143-MHz processor as well as a Sun Enterprise 3000 server with 1 GB RAM and UltraSparc-II $250-\mathrm{MHz}$ processor. ${ }^{10}$ The code developed inhouse was written in $\mathrm{C}_{++}$; compiling options were given by $-O$ - fast-DSYSSPARC. The operating system was Solaris 2.5.1, and linear programs were solved by the primal simplex algorithm of CPLEX 6.0 with an optimality tolerance parameter of $1 e^{-9}$ (cf. CPLEX [6], pp. 135, 292). As to other parameters of the primal simplex routine and the presolver (including the aggregator) of CPLEX, we changed the default setting to a more adequate setting providing faster CPU times for solving single LPs. This latter parameter setting was used for both the 'Traditional Approach' and the 'Progressive Refinement Approach' stated in sections 2.2 and 3 , respectively; i.e. the same parameter setting was utilized for solving $L P_{1}^{\nu}$ within the traditional approach as well as for solving all $L P_{1}^{\nu}$ and $L P_{2, s}^{\nu}$ within the PRA. The dynamics of the interest rates are generated by the so-called key rate model with either 2 or 3 key rates. For details of the underlying stochastic processes, the reader is referred to Frauendorfer and Haarbrücker [10].

Measurement of all run times - which are explained in detail in the following was done using the $\mathrm{C}_{++}$structure RWTimer. Even net solution times within CPLEX (like e.g. CPU time of the pure simplex algorithm including preprocess-

\footnotetext{
${ }^{10}$ In the following tables, we use the abbreviations 'WS' and 'SV' denoting 'workstation' and 'server'. According to SPEC (Standard Performance Evaluation Corporation), the performance indicator SPECfp95 of the workstation and the server has an order of magnitude of 8 and 15 , respectively.
} 
ing) were taken in this way, because operating the CPLEX 6.0 package via the so-called Callable Library interface does not support the provision of internal elapsed CPU times. Anyway, since start and stop of run-time measurement always took place immediately before and after the call of the simplex algorithm procedure, resulting measurement deviations should be negligible. Unfortunately, one has to be aware of a certain drawback of RWTimer: as it is based on the system-dependent function clock (which has an upper limit of roundabout 35 minutes on the platform used), run times exceeding this limit normally result in arbitrary behaviour of all timers and lead to useless time measurement. ${ }^{11}$ Before dealing with our - rather pragmatic - way of overcoming this drawback in Rem. 6.1, the following list provides a detailed overview of all CPU times measured; 'C.' abbreviates 'Column'.

\section{Traditional Approach}

C. 1: "All refined LPs (gross)" stands for the total time required to "solve' all refined first-stage $\operatorname{LPs} L P^{1, \nu}(\nu \geq 1)$. Here, by 'solving' we understand the execution of all the following activities: transformation of the underlying scenario tree $B^{1, \nu}$ into a LP-format accepted by CPLEX, conveying of the LP-instance to the CPLEX-Solver, solving the instance by means of the primal simplex algorithm and exporting solution information into the scenario tree.

C. 2: "All refined LPs (primal Simplex)" specifies the cumulated time spent by the primal simplex algorithm in order to solve all those LPs mentioned in column 1 .

\section{Progressive Refinement Approach}

C. 1: "Optimality Check (gross)" indicates that time which elapsed for executing all the following activities: 'solving' (see above) all necessary second-stage LPs $L P_{s}^{2, \nu}(\nu \geq 1)$, upgrading the (sub-)gradient, building up and solving the small LP within the optimality check (recall Rem. $3.2(i)$ ), and 'solving' - if necessary - refined first-stage LPs $L P^{1, \nu}$.

C. 2: "Second-Stage LPs (primal Simplex)" stands for the cumulated time spent by the primal simplex algorithm in order to solve all those LPs mentioned in column 1.

\footnotetext{
${ }^{11}$ The server and all workstations within the authors' LAN are single-processor machines always running in multi-user mode. Hence, measuring run times by means of the internal system clock - thus leading to elapsed-time values only - would have produced run times possibly far away from the real CPU times of the process under consideration. For this reason, we put up with the mentioned drawback of RWTimer.
} 
C. 3: "Total Run Time" states the total time of the PRA required to deal with the whole sequence of refined LPs up to the predefined number of refinement steps.

Please take note of the fact that a hot-start technique - i.e. taking advantage of a basis previously determined - is only applied to the PRA, but not to the traditional approach: further details justifying this different proceeding are given in Rem. 6.2.

All run times will be noted in CPU seconds. The really important CPU times are those given in the respective first column because these times record the effects of the conceptual difference between the two approaches under consideration: how much time is required - on balance - for executing the comparable steps 2-3 and 2-5 within the traditional approach of section 2.2 and the new approach of section 3, respectively? All listings of CPU times take place in a separate manner according to upper and lower approximations; for both types of approximation the so-called speedup factor is determined: speedup is computed by dividing the longer absolute run time $(\mathrm{L})$ by the shorter absolute run time $(\mathrm{S})$, i.e.

$$
\text { speedup }:=\frac{L}{S}=\frac{1}{1-\frac{L-S}{L}} \widehat{=} \frac{1}{1-(\text { relative economy of CPU time })} .
$$

(As the initial unrefined problem $L P^{1,0}$ has to be solved in both approaches in exactly the same way, the time required for solving this problem is left out of account.) Other run times serve for information purposes only and give an insight into the computational effort required for the pure simplex algorithm; additionally, these run times reveal the ratio of total run time of the PRA to merely pure times within the traditional approach, like net primal simplex time (Col. 2) or the time for 'solving' (Col. 1).

\section{Remark 6.1 :}

For ease of notation, the above-mentioned difficulty concerning the clock function limit is handled in the following way: exceeding a time cap of $2000 \mathrm{CPU}$ seconds - before having reached the predefined number of refinement steps - will be indicated by the term '> 2000'; additionally we state the refinement number at which the cap was exceeded using the term '(ref. $\nu$ )'. When, in fact, it would have been possible to master a predefined number of refinements by the particular hardware platform, we project the net simplex time in the following way: the last realized simplex time is multiplied by the number of refinements for which a correct time could not be observed; thus, we obtain a lower time bound written as ' $>$ wxyz'. In the opposite case (i.e. problems are too large to be solved on the hardware platform) we specify the primal simplex time that has elapsed until the formerly mentioned refinement step $\nu$; furthermore, we again use the notation '(ref. $\nu$ )'. Depending on the number of refinements successfully mastered before reaching the time cap of 2000 seconds, the lower time bounds can vastly underestimate 
the true CPU time: when listing the speedup, we take this fact into account using the prefix ' $>$ ' or ' $\gg$ '.

\subsection{Funding Problem Results}

All run-time results within the funding problem are based on a test set of four money market input constellations (in short MM1-MM4) given as combinations of two initial interest rate curves and two structures of maturing maturities. MM1 is composed of the real maturing structure of a major Swiss bank in June 1999 as well as the then current money market interest rate curve (having a normal structure), whereas MM2 consists of a constant maturing structure and a fictitious inverse interest rate curve. The problem instances MM3 and MM4 are formed by combining the interest rate curve of MM1 with the maturing structure of MM2 and vice versa, respectively. Altogether, the four input characteristics are stated in Table 2 ('M' is short for 'month'). Two neighbouring stages represent a

Interest Rates (\% p.a.)

\begin{tabular}{|l|c|c|c|c|c|}
\hline Maturity & $1 \mathrm{M}$ & $2 \mathrm{M}$ & $3 \mathrm{M}$ & $6 \mathrm{M}$ & $12 \mathrm{M}$ \\
\hline \hline MM1 & 0.86 & 0.91 & 0.96 & 1.06 & 1.33 \\
\hline MM2 & 1.22 & 1.20 & 1.18 & 1.12 & 1.00 \\
\hline MM3 & 0.86 & 0.91 & 0.96 & 1.06 & 1.33 \\
\hline MM4 & 1.22 & 1.20 & 1.18 & 1.12 & 1.00 \\
\hline
\end{tabular}

Maturing Volumes

\begin{tabular}{|l|l|}
\hline MM1 & (n.a.) $^{12}$ \\
\hline MM2 & constant 200'000 per period $^{\prime}$ \\
\hline MM3 & constant 200'000 per period \\
\hline MM4 & (n.a.) \\
\hline
\end{tabular}

Table 2: Initial input within the funding problem

real life period of one month, the set $\mathcal{D}$ is given by $\{1, \ldots, 12\}$, and the subset of maturities for which the money market is sufficiently liquid is defined to be $\mathcal{D}^{l i q}:=$ $\{1,2,3,6,12\}$; the maturities $1 \mathrm{M} / 12 \mathrm{M}$ and $1 \mathrm{M} / 6 \mathrm{M} / 12 \mathrm{M}$ serve as key rates for $\operatorname{dim} \Theta_{t}=K_{t}=2$ and $\operatorname{dim} \Theta_{t}=3$, respectively. Underlying Wiener processes driving the stochastic evolution of interest rates and total funding volume are taken as the same throughout all situations MM1-MM4.

Table 3 lists exemplary run-time results with $H=4$ and $\operatorname{dim} \Theta_{t}=2,3$ for MM1. Concerning the dimension of the unrefined problem $L P^{1,0}$, the maximum number of refinements was set to 250 and 200, respectively. On the basis of the speedup values, one observes a significant acceleration w.r.t. run time when using

\footnotetext{
${ }^{12}$ For reasons of confidentiality we omit the explicit structure.
} 
$\boldsymbol{H}=4, \operatorname{dim} \Theta_{t}=2,250$ Refinements

\begin{tabular}{|c||r|r|r|}
\hline $\begin{array}{c}\text { Traditional } \\
\text { Approach }\end{array}$ & $\begin{array}{c}\text { All refined LPs } \\
\text { (gross) }\end{array}$ & $\begin{array}{c}\text { All refined LPs } \\
\text { (primal Simplex) }\end{array}$ & \multirow{2}{*}{} \\
\cline { 1 - 2 } Upper Appr. & $>2000$ (Ref. 225) & $>982$ & \\
\hline Lower Appr. & $>2000$ (Ref. 125) & $>2343$ & \\
\hline \hline PRA & $\begin{array}{c}\text { Optimality Check } \\
\text { (gross) }\end{array}$ & $\begin{array}{c}\text { Second-Stage LPs } \\
\text { (primal Simplex) }\end{array}$ & $\begin{array}{c}\text { Total } \\
\text { Run Time }\end{array}$ \\
\hline Upper Appr. & 22.79 & 1.69 & 144.68 \\
\hline Lower Appr. & 57.33 & 5.09 & 344.91 \\
\hline \hline Speedup & & & \\
\cline { 1 - 2 } Upper Appr. & $>87.7$ & & \\
\cline { 1 - 2 } Lower Appr. & $\gg 34.8$ & & \\
\hline
\end{tabular}

$H=4, \operatorname{dim} \Theta_{t}=3,200$ Refinements

\begin{tabular}{|c||r|r|r|}
\hline $\begin{array}{c}\text { Traditional } \\
\text { Approach }\end{array}$ & $\begin{array}{c}\text { All refined LPs } \\
\text { (gross) }\end{array}$ & $\begin{array}{c}\text { All refined LPs } \\
\text { (primal Simplex) }\end{array}$ & \\
\cline { 1 - 2 } Upper Appr. & 1508.64 & 584.79 & \\
\cline { 1 - 2 } Lower Appr. & $>2000$ (Ref. 78) & $>3143$ & \\
\hline \hline PRA & $\begin{array}{c}\text { Optimality Check } \\
\text { (gross) }\end{array}$ & $\begin{array}{c}\text { Second-Stage LPs } \\
\text { (primal Simplex) }\end{array}$ & $\begin{array}{c}\text { Total } \\
\text { Run Time }\end{array}$ \\
\cline { 1 - 2 } Upper Appr. & 18.25 & 1.50 & 107.17 \\
\hline Lower Appr. & 96.15 & 10.58 & 485.36 \\
\hline \hline Speedup & & & \\
\cline { 1 - 2 } Upper Appr. & 82.7 & & \\
\hline Lower Appr. & $\gg 20.8$ & & \\
\cline { 1 - 2 }
\end{tabular}

Table 3: Comparison of both approaches within MM1 (WS)

the PRA. Outperformance is particularly high concerning the upper approximations: one achieves speedups of approximately 80. As to the lower approximations, the outperformance is of a somewhat lower order of magnitude, but nonetheless in no way negligible. This last fact is indeed caused by the time cap of 2000 seconds: normally, the lower approximations would rather be predestined to benefit more from the PRA than the upper approximations because of the much larger size of LP instances; but since the traditional approach mostly exceeds the time cap already after very few refinements, much smaller speedup values are observed.

Hence, indicated speedups sometimes considerably underestimate the true run-time acceleration, which will be stressed by preceding the values with the symbols ' $\gg$ ' or ' $>$ ' (depending on the number of successfully handled refinement steps before reaching the time cap). Obviously, the much better run-time behaviour of the PRA is based on the low - and constant! - dimension of all 


\begin{tabular}{|c|c|c|c|c|c|}
\hline \multicolumn{3}{|c|}{ Funding Problem } & Upper Appr. & Lower Appr. & $\begin{array}{r}\text { Weighted } \\
\text { Average }\end{array}$ \\
\hline \multirow{4}{*}{ MM1 } & \multirow{2}{*}{$H=4$} & $\operatorname{dim} \Theta_{t}=2$ & 87.7 & 34.8 & $\overline{61.2}$ \\
\hline & & $\operatorname{dim} \Theta_{t}=3$ & 82.6 & 20.8 & 47.3 \\
\hline & \multirow{2}{*}{$H=6$} & $\operatorname{dim} \Theta_{t}=2$ & 53.4 & 11.2 & 29.1 \\
\hline & & $\operatorname{dim} \Theta_{t}=3$ & 52.6 & 2.8 & 24.0 \\
\hline \multirow{4}{*}{ MM2 } & \multirow{2}{*}{$H=4$} & $\operatorname{dim} \Theta_{t}=2$ & 85.5 & 13.3 & 49.4 \\
\hline & & $\operatorname{dim} \Theta_{t}=3$ & 80.3 & 20.8 & 46.5 \\
\hline & \multirow{2}{*}{$H=6$} & $\operatorname{dim} \Theta_{t}=2$ & 52.7 & 2.6 & 24.1 \\
\hline & & $\operatorname{dim} \Theta_{t}=3$ & 51.9 & 2.1 & 23.4 \\
\hline \multirow{4}{*}{ MM3 } & \multirow{2}{*}{$H=4$} & $\operatorname{dim} \Theta_{t}=2$ & 87.7 & 35.1 & 61.4 \\
\hline & & $\operatorname{dim} \Theta_{t}=3$ & 82.5 & 20.6 & 47.3 \\
\hline & \multirow{2}{*}{$H=6$} & $\operatorname{dim} \Theta_{t}=2$ & 53.1 & 11.3 & 29.1 \\
\hline & & $\operatorname{dim} \Theta_{t}=3$ & 53.2 & 2.8 & 24.3 \\
\hline \multirow{4}{*}{ MM4 } & \multirow{2}{*}{$H=4$} & $\operatorname{dim} \Theta_{t}=2$ & 87.9 & 13.0 & 50.4 \\
\hline & & $\operatorname{dim} \Theta_{t}=3$ & 82.2 & 20.8 & 47.3 \\
\hline & \multirow{2}{*}{$H=6$} & $\operatorname{dim} \Theta_{t}=2$ & 53.4 & 2.6 & 24.4 \\
\hline & & $\operatorname{dim} \Theta_{t}=3$ & 52.6 & 2.1 & 23.8 \\
\hline \multicolumn{3}{|c|}{$\overline{\bar{\phi}}$} & "68.7 & 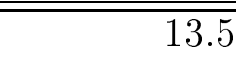 & 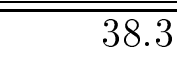 \\
\hline
\end{tabular}

Table 4: Minimum speedup for MM1 - MM4 (WS)

second-stage LPs $L P_{s}^{2, \nu}$, contrary to the expanding refined instances $L P^{1, \nu}$ : this is shown in the very much shorter CPU times required by the simplex algorithm itself and applies to both the lower and upper approximations.

We would like to point out that the total run time of the executable - when using the PRA - takes only approximately $15 \%$ - $20 \%$ of the net simplex algorithm time within the traditional approach. This underlines the superior performance of the new approach.

For shortage of space, a detailed presentation of run-time results is omitted for the problems MM2-MM4 as well as for an analogous setting with $H=6$ and 80 refinements. Instead, we summarize all observed speedups for $H=4,6$ of MM1-MM4 in the first two columns of Table $4 .{ }^{13}$ Furthermore, the third column indicates weighted averages of the speedups for the lower and upper approximations; here, the weights are given by the absolute benchmark run times (i.e. the gross run times within the traditional approach for solving all refined LPs or the time cap 2000, respectively). Altogether, one observes minimum average speedups of approx. 68 and 13 w.r.t. upper and lower approximations, and an overall weighted average speedup of 38 . Hence, the new PRA outperforms the

\footnotetext{
${ }^{13}$ It should be repeated that the specified speedups are - sometimes extreme - underestimations of the true acceleration.
} 
$H=8, \operatorname{dim} \Theta_{t}=2,80$ Refinements

\begin{tabular}{|c|c|c|c|}
\hline $\begin{array}{l}\text { Traditional } \\
\text { Approach }\end{array}$ & $\begin{array}{l}\text { All refined LPs } \\
\text { (gross) }\end{array}$ & $\begin{array}{c}\text { All refined LPs } \\
\text { (primal Simplex) }\end{array}$ & \\
\hline Upper Appr. & $>2000$ (Ref. 47) & 541.98 (Ref. 47) & \\
\hline Lower Appr. & $>2000$ (Ref. 8) & 260.27 (Ref. 8) & \\
\hline PRA & $\begin{array}{c}\text { Optimality Check } \\
\text { (gross) }\end{array}$ & $\begin{array}{c}\text { Second-Stage LPs } \\
\text { (primal Simplex) }\end{array}$ & $\begin{array}{c}\text { Total } \\
\text { Run Time }\end{array}$ \\
\hline Upper Appr. & 73.35 & 5.99 & 231.95 \\
\hline Lower Appr. & 1152.54 & 169.67 & ca. 2946 \\
\hline \multicolumn{4}{|l|}{$\overline{\text { Speedup }}$} \\
\hline Upper Appr. & $\gg 27.2$ & & \\
\hline Lower Appr. & $\gg 1.7$ & & \\
\hline
\end{tabular}

$H=8, \operatorname{dim} \Theta_{t}=3,80$ Refinements

\begin{tabular}{|c|c|c|c|}
\hline $\begin{array}{l}\text { Traditional } \\
\text { Approach }\end{array}$ & $\begin{array}{l}\text { All refined LPs } \\
\text { (gross) }\end{array}$ & $\begin{array}{c}\text { All refined LPs } \\
\text { (primal Simplex) }\end{array}$ & \\
\hline Upper Appr. & $>2000$ (Ref. 46) & 492.08 (Ref. 46) & \\
\hline Lower Appr. & 1431.26 (Ref. 1) & 113.27 (Ref. 1) & \\
\hline PRA & $\begin{array}{c}\text { Optimality Check } \\
\text { (gross) }\end{array}$ & $\begin{array}{c}\text { Second-Stage LPs } \\
\text { (primal Simplex) }\end{array}$ & $\begin{array}{c}\text { Total } \\
\text { Run Time }\end{array}$ \\
\hline Upper Appr. & 74.49 & 5.65 & 244.69 \\
\hline Lower Appr. & 1256.86 (Ref. 11) & 303.91 (Ref. 11) & $\begin{array}{r}>2000 \\
\text { (Ref. 11) }\end{array}$ \\
\hline \multicolumn{4}{|l|}{ Speedup } \\
\hline Upper Appr. & $\gg 26.8$ & & \\
\hline Lower Appr. & (n.a.) & & \\
\hline
\end{tabular}

Table 5: Comparison of both approaches within MM1 - Sequel II (SV)

traditional approach in an impressive manner for time horizons $H=4,6$.

Regarding longer time horizons, the outperformance by the PRA remains still valid - at least concerning the upper approximations. Table 5 presents run times and speedups for MM1 with $H=8$, but this time they have been determined on the server. All computation runs using the traditional approach were interrupted before having reached the 80 refinements called for: at the latest, after 47 refinement steps, the cap of 2000 seconds was exceeded. On the contrary, the PRA proved satisfactory concerning all upper approximations and could successfully handle 80 refinements. Moreover, both the gross run times for the optimality check and the total run time ranged between acceptable limits so that minimum speedups of 27.2 and 26.8 for $\operatorname{dim} \Theta_{t}=2$ and $\operatorname{dim} \Theta_{t}=3$ could be achieved.

Admittedly, concerning approximations from below, even the PRA relatively 


\begin{tabular}{|l|l|l||r|}
\hline \multicolumn{3}{|c|}{ Funding Problem } & Upper Appr. \\
\hline \hline \multirow{2}{*}{ MM1 } & \multirow{2}{*}{$H=8$} & $\operatorname{dim} \Theta_{t}=2$ & 27.2 \\
\cline { 3 - 4 } & & $\operatorname{dim} \Theta_{t}=3$ & 26.8 \\
\hline \multirow{2}{*}{ MM2 } & \multirow{2}{*}{$H=8$} & $\operatorname{dim} \Theta_{t}=2$ & 26.4 \\
\cline { 3 - 4 } & & $\operatorname{dim} \Theta_{t}=3$ & 26.2 \\
\hline \multirow{2}{*}{ MM3 } & \multirow{2}{*}{$H=8$} & $\operatorname{dim} \Theta_{t}=2$ & 27.1 \\
\cline { 3 - 4 } & & $\operatorname{dim} \Theta_{t}=3$ & 23.0 \\
\hline \multirow{2}{*}{ MM4 } & \multirow{2}{*}{$H=8$} & $\operatorname{dim} \Theta_{t}=2$ & 26.3 \\
\cline { 3 - 4 } & & $\operatorname{dim} \Theta_{t}=3$ & 26.0 \\
\hline \hline \multicolumn{3}{|c|}{$\varnothing$} \\
\hline
\end{tabular}

Table 6: Minimum speedup for MM1 to MM4 - Sequel (SV)

soon exceeds the limit of manageable problem dimensions unless the number of stochastic dimensions influencing the interest rate dynamics is kept rather small: though 80 refinements could successfully be managed for $\operatorname{dim} \Theta_{t}=2$, the total run time soon reached an order of magnitude of approx. 3000 seconds; and already at $\operatorname{dim} \Theta_{t}=3$, the so-called curse of dimensionality leads to problem sizes which are no longer manageable, even within the PRA; hence, we forego reporting speedups w.r.t. lower approximations. Similar detailed results for MM2-MM4 are left out, and we restrict ourselves in Table 6 to the presentation of speedups only. (Just the upper approximations are considered because of the above mentioned aspects.) One realizes a minimum average speedup of 26 , still demonstrating significant outperformance with regard to upper approximations.

The following remark deals with the feature of the CPLEX package to apply a hot-start technique for solving single LPs.

\section{Remark 6.2 :}

(i) As already stated at the beginning of subsection 2.2, the fact that all secondstage subproblems $L P_{2, s}^{\nu}$ are of exactly the same size is important for the good performance of the PRA. Since the subproblems are all equally sized, an internal CPLEX feature can be used taking advantage of an advanced basis: once having solved a subproblem and written the corresponding optimal basis to a file, one can read in this file before solving the next subproblem so that the primal simplex algorithm routine uses this basis for a hot start. In our case, this leads to a reduced CPU time for solving a single subproblem LP; this is particularly advantageous when step 2 of the 'Progressive Refinement Approach' in section 3 has to be performed, because then a possibly large number of equally-sized subproblems must be solved.

(ii) CPLEX would also allow the reading of the basis of an even smaller problem and using this as an advanced basis when solving a larger problem. 
Hence, although in the sequence of refined LPs $L P^{1, \nu}$ the problem instances are never of the same dimension, applying the hot-start technique could nonetheless be profitable because "even if the problem is significantly different, using an advanced basis may still help" (see CPLEX [6], p.30). However, tests by the authors have shown nonuniform results within the traditional approach when using advanced bases: on the one hand, in some cases the net simplex time was reduced; on the other hand, this reduction was nearly annihilated when taking the gross CPU time of 'solving' all refined first-stage LPs (i.e. reading and saving the basis files almost compensated the speedup of the pure simplex algorithm). But there were even cases where simplex algorithm performed poorer with hot-start technique than without: e.g. for a setting of $H=6, \operatorname{dim} \Theta_{t}=3$, lower approximation, the net simplex algorithm CPU time with hot-start technique more than tripled compared to without hot-start technique for the same number of refinements. (A more detailed analysis on this aspect can be found in Haarbrücker [14], pp. 120ff.) This led to the conclusion, that the hot-start technique is only favourable for some of the test problems under consideration: as it is aimed to compare the traditional approach and the PRA in a general manner, we forego a 'trimming' of the traditional approach to special test problems' subclasses and generally disregard advanced bases within the traditional approach.

Table 3 reveals that the predefined maximum number of refinements - which can be managed within the time period of $2000 \mathrm{sec}$ - - is always determined by the time-consuming benchmark runs, i.e. by the traditional approach. In order to get an insight into the computational limits of the PRA for shorter time horizons $H=4,6$, we reran the same computations on the workstation: but now only using the PRA, and the number of refinements to be handled was set much higher than before.

A representative extract of the observed results concerning medium-sized problems $H=4, \operatorname{dim} \Theta_{t}=3$ and $H=6, \operatorname{dim} \Theta_{t}=2$ is summarized in Table 7 . Columns $2-5$ denote the number of refinements as well as the known three types of measured run times. Since it was aimed at reaching the same refinement state for all problems MM1-MM4, the formerly fixed cap of 2000 sec. is now only to be seen as coarse index. If total run time could be read exactly or if it could be approximated (despite having exceeded the cap), then the exact or the approximate time are denoted, respectively; otherwise, the notation ' $\gg 2000$ ' stands for a run time unable to be extrapolated and far above the cap. This latter case occurs when the optimality check is 'negative' at an advanced stage of the refinement process; negative in the sense that the formerly achieved optimal decision could not be verified to be still optimal after the very last refinement step. In the last two columns, both the number of such negative optimality checks and the 
actually occurring changes in today's optimal decision $x^{1}$ are reported. As supplementary information, the indices of those refinement steps where a negative optimality check took place are indicated in brackets. After careful consideration of the results listed in Table 7, the following statements can be made:

(a) Only very rarely, optimality checks occur which do not prove a former decision to be still optimal. But in the seldom cases where they occur, one has to solve a refined LP as a whole at an advanced stage of the refinement process; this will yield a massive increase of run time as can be seen regarding the lower approximations in MM2 and MM3. Obviously, in such cases the PRA is as heavily confronted with the curse of dimensionality as in the traditional one.

(b) All observed cases of negative optimality checks were in fact followed by a switch in today's optimal decision $x^{1}$, i.e. the relaxation of the normal cone (according to the comments following (19)) did not really lead to undesirable side-effects. ${ }^{14}$

(c) Usually, i.e. without being faced with negative optimality checks, numerous refinement steps are manageable with a suitable time exposure. Of course, even within the PRA one is faced with expanding scenario trees. Hence, the problem dimension ( $H, \operatorname{dim} \Theta_{t}$, type of approximation) still influences strongly the number of refinements which can be dealt with in a fixed period of time.

(d) CPU time required by the pure simplex algorithm makes up a very small part (approx. $5-10 \%$ ) of the gross time needed for the optimality check. The latter in turn forms just a small part - particularly concerning upper approximations - of the total run time. Hence, unless case (a) eventuates, the bottleneck in solving a sequence of approximating refined MSLP does not lie any longer in the solution activity by a direct method but rather in preparatory operations like the generation or the refinement of scenario trees.

\section{Conclusions and Outlook}

The point of departure was given by a multistage stochastic linear program in a rather general form. Provided that the multistage stochastic program is to be solved using a scenario-based method leading to expanding scenario trees, we introduced a procedure aiming at faster solving of a sequence of large-scale LPs associated with those scenario trees. If both the scenario generation technique

\footnotetext{
${ }^{14}$ However, the occurence of zeros or very small maturing volumes in the initial structure of maturing volumes can (in conjunction with specific initial interest rate curves) result in negative optimality checks without a subsequent switch of $x^{1}$. In this case, the relaxation of the normal cone has possibly to be critized as problematic.
} 
$H=4, \operatorname{dim} \Theta_{t}=3$

\begin{tabular}{|c|l||r|r|r|r||r|c|}
\hline Problem & Approx. & \# Ref. & $\begin{array}{c}\text { Optim. Check } \\
\text { (gross) }\end{array}$ & $\begin{array}{c}\text { Second-Stage LPs } \\
\text { (primal Simplex) }\end{array}$ & $\begin{array}{c}\text { Run Time } \\
\text { total }\end{array}$ & $\begin{array}{c}\text { \# negative } \\
\text { Optim. Check }\end{array}$ & $\begin{array}{c}\text { \# Changes } \\
\text { in } x^{1}\end{array}$ \\
\hline \hline \multirow{2}{*}{ MM1 } & Upper & 850 & 78.63 & 6.38 & 2053.22 & 0 & 0 \\
\cline { 2 - 8 } & Lower & 400 & 194.84 & 20.48 & 1929.73 & 0 & 0 \\
\hline \multirow{2}{*}{ MM2 } & Upper & 850 & 79.67 & 7.43 & 2092.25 & 0 & 0 \\
\cline { 2 - 8 } & Lower & 400 & (n.a.) & 29.49 & $\gg 2000$ & $1(373)$ & 1 \\
\hline \multirow{2}{*}{ MM3 } & Upper & 850 & 80.53 & 6.49 & 2061.64 & 0 & 0 \\
\cline { 2 - 8 } & Lower & 400 & 196.85 & 20.53 & 1950.05 & 0 & 0 \\
\hline \multirow{2}{*}{ MM4 } & Upper & 850 & 80.22 & 6.74 & 2050.57 & 0 & 0 \\
\cline { 2 - 8 } & Lower & 400 & (n.a.) & 31.05 & $\gg 2000$ & $1(373)$ & 1 \\
\hline
\end{tabular}

$H=6, \operatorname{dim} \Theta_{t}=2$

\begin{tabular}{|c|c||r|r|r|r||c|c|}
\hline \multirow{2}{*}{ Problem } & Approx. & \# Ref. & $\begin{array}{c}\text { Optim. Check } \\
\text { (gross) }\end{array}$ & $\begin{array}{c}\text { Second-Stage LPs } \\
\text { (primal Simplex) }\end{array}$ & $\begin{array}{c}\text { Run Time } \\
\text { total }\end{array}$ & $\begin{array}{c}\text { \# negative } \\
\text { Optim. Check }\end{array}$ & $\begin{array}{c}\text { \# Changes } \\
\text { in } x^{1}\end{array}$ \\
\hline \hline \multirow{2}{*}{ MM1 } & Upper & 450 & 160.54 & 11.43 & ca. 2152 & 0 & 0 \\
\cline { 2 - 8 } & Lower & 180 & 401.61 & 40.56 & 1966.73 & 0 & 0 \\
\hline \multirow{2}{*}{ MM2 } & Upper & 450 & 161.74 & 13.45 & ca. 2190 & 0 & 0 \\
\cline { 2 - 8 } & Lower & 180 & 827.24 & 46.47 & ca. 2394 & $1(39)$ & 1 \\
\hline \multirow{2}{*}{ MM3 } & Upper & 450 & 162.50 & 11.85 & ca. 2213 & 0 & 0 \\
\cline { 2 - 8 } & Lower & 180 & 399.96 & 40.61 & 1998.54 & 0 & 0 \\
\hline \multirow{2}{*}{ MM4 } & Upper & 450 & 162.33 & 12.98 & 2136.30 & 0 & 0 \\
\cline { 2 - 8 } & Lower & 180 & 833.45 & 46.24 & ca. 2404 & $1(39)$ & 1 \\
\hline
\end{tabular}

Table 7: Numerous refinements of funding problems within the PRA (WS) 
utilized and the problem to be solved fulfil certain requirements (see below), it was expected that the presented progressive refinement approach would accelerate the computations in the sense of CPU time reduction.

As a validation, a financial problem situation - within the area of running fixed income products - was stated in section 4. Various computation runs took into account miscellaneous time horizons, stochastic dimensions and numbers of refinements, and were computed on two different hardware environments. In all cases, the results w.r.t. elapsed CPU time are promising in the sense that a significant speedup could be achieved. As a consequence thereof, really numerous refinements (up to several hundred) can be managed successfully with adequate time exposure - quite contrary to the traditional approach, which is heavily restricted w.r.t. practical application.

In general, two facts are indispensable for the success of the new approach: first, the refinement technique used has to ensure that only small parts of the scenario tree are modified whereas the main parts of the tree remain totally unchanged; secondly, the multistage stochastic problem must distinguish itself by a robust root node decision, i.e. the decision remains constant over longer sequences of refinement steps. As could be seen, there are real-life problem situations characterized by this special attribute: e.g., the stated funding problem where successful results could be achieved when operated in cooperation with a major Swiss bank.

Since a thorough treatment of the accuracy of the approximations would be outside the scope of this paper, we will only state some aspects in brief: the PRA allows for numerous refinements when applying the Barycentric Approximation technique, but the approximation accuracy ${ }^{15}$ can only be improved up to a certain degree as long as refinement is restricted to the root node only. It is planned to treat a detailed presentation of accuracy values in a subsequent paper. Although refinement in the root node increases the number of scenarios as much as possible (compared with refinement at other stages), this is no guarantee of a significant improvement of the accuracy of approximation. In order to achieve an acceptable accuracy, one therefore has to think about an extension of the new approach which is implementable for refinements at other stages than the root node, too.

In addition to the above-mentioned extension, several further topics offer themselves for future investigation. First, on the operational level, it remains to be studied if the PRA provides a similar outperformance when applying other discretization techniques than the barycentric one. Second, on the theoretical level, there is the challenge of overcoming the rather pragmatic partial relaxation of the normal cone (according to the remarks after (19)): is there any smart way to determine the feasibility set $K^{2}$ introduced in (16) and to exploit $\mathcal{N}_{K^{2}}$ within the optimality check as an additive part of the entire normal cone?

Lastly, it could be worthwhile investigating whether a more appropriate model

\footnotetext{
${ }^{15}$ defined by Accuracy $:=\frac{\text { Obj.-Value of Upper Appr. - Obj.-Value of Lower Appr. }}{\text { Obj.-Value of Lower Appr. }}$
} 
formulation or another shape of the arising large-scale LPs is able to reduce the (sometimes substantial) numerical effort of the preprocessing within the Simplex method - a question particularly aimed at the specific formal model in section 4 and the CPLEX package).

\section{References}

[1] Birge, J.R. Current Trends in Stochastic Programming Computation and Applications. Working Paper, Department of Industrial and Operations Engineering, University of Michigan, 1995.

[2] Birge, J.R., Louveaux, F. Introduction to Stochastic Programming. Springer-Verlag, New York, 1997.

[3] Birge, J.R., QI, L. Continuous approximation schemes for stochastic progams. Annals of Operations Research 56 (1995), 15-38.

[4] Birge, J.R., Wallace, S.W. A separable piecewise linear upper bound for stochastic linear programs. SIAM Journal on Control and Optimization 26 (1988), 725-739.

[5] Birge, J.R., Wets, R.J.-B. Sublinear upper bounds for stochastic programs with recourse. Mathematical Programming 43 (1989), 131-149.

[6] CPLEX Documentation. Version 5.0 of the CPLEX Callable Library. ILOG Inc., CPLEX Division, Incline Village, USA, 1997.

[7] Frauendorfer, K. Stochastic Two-Stage Programming. Lecture Notes in Economics and Mathematical Systems, No. 392. Springer-Verlag, Berlin, 1992.

[8] Frauendorfer, K. Multistage stochastic programming: Error analysis for the convex case. Z. Oper. Res. 39:1 (1994), 93-122.

[9] Frauendorfer, K. Barycentric scenario trees in convex multistage stochastic programming. Mathematical Programming 75:2 (1996), 277-294.

[10] Frauendorfer, K., Haarbrücker, G. Test Problems in Stochastic Multistage Programming. Optimization 47:3-4 (2000), 267-285.

[11] Frauendorfer, K., Marohn, C. Refinement Issues in Stochastic Multistage Linear Programming. In Stochastic Programming Methods and Technical Applications (Proceedings of the 3rd GAMM/IFIP Workshop 1996), Lecture Notes in Economics and Mathematical Systems, No. 458 (1998), Marti, K., Kall, P., Ed., Springer-Verlag, Berlin, pp. 305-328. 
[12] Gaese, R. Risk Management and Risk Measurement. Ph.D. Thesis No. 2195, University of St. Gallen, 1999.

[13] HaArbrücker, G. Generalized Barycenters of Cross Simplices: An Illustrative Approach. Working Paper WP gh-07/1999-02, Institute of Operations Research, University of St. Gallen (1999).

[14] HaArbrüCKer, G. Sequentielle Optimierung verfeinerter Approximationen in der mehrstufigen stochastischen linearen Programmierung. Doctoral thesis No. 2410, University of St. Gallen, 2000.

[15] Kall, P., Ruszczyński, A.,Frauendorfer, K. Approximations in Stochastic Programming. In Numerical Techniques for Stochastic Optimization (1988), Ermoliev, Yu., Wets, R. J.-B., Ed., Springer Series in Computational Mathematics, vol. 10, pp. 33-64.

[16] Kall, P., Wallace, S.W. Stochastic Programming. Wiley, Chichester, 1994.

[17] Marohn, C. Stochastische mehrstufige lineare Programmierung im Asset \& Liability Management. Bank- und finanzwirtschaftliche Forschungen, Bd. 282. Paul Haupt Verlag, Bern, 1998.

[18] Rockafellar, R. Convex Analysis. Princeton Landmarks in Mathematics. Princeton University Press, Princeton, 1997.

[19] Ruszczyński, A. Some advances in decomposition methods for stochastic linear programming. Ann. Oper. Res. 85 (1999), 153-172.

[20] Ruszczyński, A., Swietanowski, A. Accelerating the regularized decomposition method for two stage stochastic linear problems. Eur. J. Oper. Res. 101:2 (1997), 328-342.

[21] Schürle, M. Zinsmodelle in der stochastischen Optimierung. Bank- und finanzwirtschaftliche Forschungen, Bd. 279. Paul Haupt Verlag, Bern, 1998.

[22] Wallace, S.W. Solving stochastic programs with network recourse. Networks 16 (1986), 295-317.

[23] Wets, R.J.-B. Stochastic Programs with Recourse: A Basic Theorem for Multistage Problems. Z. Wahrscheinlichkeitstheorie verw. Geb. 21 (1972), 201-206.

[24] Wets, R.J.-B. Stochastic programs with fixed recourse: The equivalent deterministic program. SIAM Rev. 16 (1974), 309-339. 
[25] Wets, R.J.-B. Stochastic Programming. In Handbooks in Operations Research and Management Science, 1: Optimization (1989), Nemhauser, G.L. et al., Ed., Elsevier Science Publishers, Amsterdam, pp. 573-629. 